\title{
MEAN FLOW AND TURBULENCE CHARACTERISTICS IN AN URBAN ROUGHNESS SUBLAYER
}

\author{
PETRA KASTNER-KLEIN ${ }^{1,2, \star}$ and MATHIAS W. ROTACH ${ }^{1}$ \\ ${ }^{1}$ Institute for Atmospheric and Climate Science, ETH Zurich, Winterthurerstrasse 190, 8057 Zurich, \\ Switzerland; ${ }^{2}$ School of Meteorology, University of Oklahoma, Norman, Oklahoma, U.S.A.
}

(Received in final form 6 May 2003)

\begin{abstract}
In this study, a detailed model of an urban landscape has been re-constructed in the wind tunnel and the flow structure inside and above the urban canopy has been investigated. Vertical profiles of all three velocity components have been measured with a Laser-Doppler velocimeter, and an extensive analysis of the measured mean flow and turbulence profiles carried out. With respect to the flow structure inside the canopy, two types of velocity profiles can be distinguished. Within street canyons, the mean wind velocities are almost zero or negative below roof level, while close to intersections or open squares, significantly higher mean velocities are observed. In the latter case, the turbulent velocities inside the canopy also tend to be higher than at street-canyon locations. For both types, turbulence kinetic energy and shear stress profiles show pronounced maxima in the flow region immediately above roof level.

Based on the experimental data, a shear-stress parameterization is proposed, in which the velocity scale, $u_{s}$, and length scale, $z_{s}$, are based on the level and magnitude of the shear stress peak value. In order to account for a flow region inside the canopy with negligible momentum transport, a shear stress displacement height, $d_{s}$, is introduced. The proposed scaling and parameterization perform well for the measured profiles and shear-stress data published in the literature.

The length scales derived from the shear-stress parameterization also allow determination of appropriate scales for the mean wind profile. The roughness length, $z_{0}$, and displacement height, $d_{0}$, can both be described as fractions of the distance, $z_{s}-d_{s}$, between the level of the shear-stress peak and the shear-stress displacement height. This result can be interpreted in such a way that the flow only feels the zone of depth $z_{s}-d_{s}$ as the roughness layer. With respect to the lower part of the canopy $\left(z<d_{s}\right)$ the flow behaves as a skimming flow. Correlations between the length scales $z_{s}$ and $d_{S}$ and morphometric parameters are discussed.

The mean wind profiles above the urban structure follow a logarithmic wind law. A combination of morphometric estimation methods for $d_{0}$ and $z_{0}$ with wind velocity measurements at a reference height, which allow calculation of the shear-stress velocity, $u_{*}$, appears to be the most reliable and easiest procedure to determine mean wind profile parameters. Inside the roughness sublayer, a local scaling approach results in good agreement between measured and predicted mean wind profiles.
\end{abstract}

Keywords: Local scaling, Street canyon, Urban meteorology, Wind profile, Wind tunnel.

\section{Introduction}

The structure of the atmospheric boundary layer above urban areas is of particular interest for air pollution modelling. In urban-scale dispersion models, the lowest portion of the boundary layer is often represented using surface-layer similarity

* Address for correspondence: School of Meteorology, University of Oklahoma, SEC 1354, 100 East Boyd, Norman, OK 73019-1013, U.S.A. E-mail: pkklein@ou.edu 
parameterizations. The urban effects are then taken into account by changes of surface roughness and heat flux. Strictly speaking, boundary-layer formulations of this type are only applicable in the inertial sublayer (ISL) well above the building tops. Inside the ISL, turbulent fluxes are approximately constant and for neutral stratification a uniform mean velocity distribution can be found (Plate, 1995), which is fairly well described by the well-known logarithmic law (in the following referred to as the log law):

$$
u(z)=\frac{u_{*}}{\kappa} \ln \left(\frac{z-d_{0}}{z_{0}}\right),
$$

with the parameters: displacement height $d_{0}$, roughness length $z_{0}$, shear-stress (friction) velocity $u_{*}$, and von Kármán constant $\kappa=0.4$. In this paper, the symbols $u, v$, and $w$ refer to mean values of the along-wind, lateral and vertical velocity components and $u^{\prime}, v^{\prime}$, and $w^{\prime}$ to the corresponding velocity fluctuations.

In the so-called roughness sublayer (RSL), i.e. the flow region in the immediate vicinity of the urban canopy elements, the flow depends locally on the particular building arrangements and has, therefore, a rather complex structure (Raupach, 1980). In the vertical, the RSL extends from the surface up to a level at which horizontal homogeneity of the flow is achieved. This occurs at 2 to 5 times the average canopy-element height (Raupach et al., 1991), and in areas with high buildings, it can occupy a significant part of the urban boundary layer (UBL) where most of the pollution problems occur. Thus, urban air pollution modelling requires also information on flow characteristics within the RSL.

An extensive review of atmospheric turbulence observations over cities has been presented by Roth (2000). However, there are only a limited number of field observations that deliver information on the mean flow and turbulence characteristics inside the urban RSL. Rotach (1993a, b, 1995) analyzed mean flow and turbulence measurements inside and above an urban street canyon. He found that one of the characteristic features of the RSL is an increase in the absolute value of Reynolds stress, from essentially zero at the average zero plane displacement height up to a maximum value, which he observed at about two times the average building height. Rotach $(1999,2001)$ concluded that the maximum value marks the level of transition from the RSL to the ISL, and that the maximum flux values are a good estimate for the friction velocity $u_{*}$.

Similar Reynolds stress profiles were observed by Oikawa and Meng (1995) at the outer edge of a suburban area (prevailing winds coming from the direction of the built-up area). The profiles peaked at a level 1.5 times the average building height $\bar{H}$. The friction velocity $u_{*}$ was determined from flux measurements at $2.6 \bar{H}$, a height that they considered to be above the RSL; this $u_{*}$ value was about $7 \%$ lower than the peak value. Feigenwinter et al. (1999), who measured the Reynolds stress at three levels $(z / \bar{H}=1.5,2.1,3.2)$ above an urban canopy under neutral conditions, also observed a significant increase of Reynolds stress between 
the two lower levels, whereas the values were almost constant between the second and third level.

Even more pronounced stress peaks were observed by Louka (1999) at a suburban site within an array of four canyon type buildings, see also Louka et al. (2000). The maximum values were found close to the building tops and were between 2 and 5 times higher than values at the highest measurement level, which was at $z / \bar{H}=2.26$. Higher peak values occurred for situations with an attributed smooth-rough transition. Like Oikawa and Meng (1995), Louka (1999) also determined $u_{*}$ from the flux measurements at the highest measurement level $(z / \bar{H}=2.26)$, which was considered to be above the RSL. The first results of an extensive urban measurement campaign (BUBBLE) in Basel, Switzerland confirm previous findings (Christen et al., 2002). Turbulent shear stress and turbulence kinetic energy peaked just above roof-level.

Although some important aspects of flow and turbulence characteristics in the urban RSL could be identified by these field studies, there are still open questions concerning appropriate scaling concepts and a lack of high resolution datasets, which cover a wider range of sampling positions and a variety of building arrangements.

Wind-tunnel modelling of flow field characteristics in the near field of obstacles is a good alternative to high-resolution datasets. There have been several windtunnel studies on flow over plant canopies, see, e.g., the review in Kaimal and Finnigan (1994) and internal boundary-layer development after a step change in surface roughness (Garratt, 1990; Pendergrass and Arya, 1984). Inside plant canopies, Reynolds stress usually strongly decreases from the value at the canopy top down to almost zero at half canopy height. Above canopy the Reynolds stress and velocity variances are almost constant with height. The internal boundary-layer studies have shown that profiles of turbulence characteristics with significant peak values can be observed in the region close to a change of surface roughness. To what extent these results are applicable to urban canopies, which are characterized by significantly larger roughness elements, higher packing densities and variable building geometry has to be further investigated.

Recently, Brown et al. (2000) investigated the flow inside and above an array of two-dimensional idealized street canyons and found that profiles with pronounced peak values of turbulence characteristics are typical for the first two canyons whereas the flow is vertically more homogenous after a fetch of 3-4 canyons. In a similar study, Rafailidis (1997) still observed pronounced peaks in Reynolds stress and turbulence intensity profiles above an array of idealized street canyons for particular roof shapes. Kastner-Klein et al. (2001) analyzed mean flow and turbulence data measured in wind-tunnel models of idealized street canyons in comparison with data from corresponding field studies, and found qualitative similarity between wind-tunnel flow characteristics and their atmospheric counterparts. Cheng and Castro (2002) presented detailed mean flow and turbulence data inside and above idealized urban surfaces, which consisted of regularly spaced (packing 
density 25\%) cubes and rectangular blocks. Configurations with uniform and variable element heights were investigated. They found that the ISL is much thinner over roughness structures with variable building heights than for structures where the height is uniform. They therefore concluded that an ISL might not exist over realistic urban surfaces with extremely heterogeneous and irregular roughness elements. They also addressed the problem of defining $u_{*}$ over rough areas and found that spatially averaged mean wind profiles inside the RSL and ISL can be best described by a single log law when $u_{*}$ is determined from direct drag measurements, or from shear stress data averaged throughout the RSL and ISL.

Additionally, a number of wind-tunnel studies have been conducted focusing on flow and dispersion patterns inside and above regular obstacle arrays (MacDonald, 1998, 2000; Theurer, 1999). However, their information on the turbulence structure in the urban RSL is rather limited. MacDonald (2000) applied a simple model that was originally derived for vegetative canopies to determine the wind velocity profile within an array of regularly spaced obstacles. The predicted exponential profiles described the spatially averaged velocity distribution within the obstacle canopy fairly well. However, the model is restricted to building configurations with low packing densities, since it is based on a simple gradient-diffusion approach. Thus it cannot cope with counter-flow vortex structures, which are typically observed in the lower part of the canopy, when the buildings occupy more than $35 \%$ of the total surface area (skimming flow regimes).

So far, wind-tunnel studies have not been published, in which high-resolution measurements of mean flow and turbulence structures were conducted inside and above a realistic urban canopy with highly variable building heights and shapes. This lack of data provided the motivation for a wind-tunnel study of the flow field in a central part of the city of Nantes in France. The experimental set-up and results of this study are described in the present paper. Based on the results, parameterization concepts for profiles of mean flow and turbulence statistics are presented, which will then also be compared with experimental data published in the literature. First, important concepts that are used in the analysis of the wind-tunnel data are summarized.

\section{Important Characteristics of the Urban Boundary Layer}

\subsection{MEAN WIND PROFILE}

As discussed in the introduction, the mean wind profile in the urban ISL can be fairly well described by the log law (Equation (1)). However, application of Equation (1) requires knowledge on the displacement height, $d_{0}$, roughness length, $z_{0}$, and friction velocity, $u_{*}$. The estimation of these parameters is not straightforward in an urban environment. The determination of $u_{*}$ is complicated by the horizontal and vertical variability of momentum fluxes inside the urban RSL. Estimates based 
on flux measurements at one sampling location do not necessarily resemble integral flow properties (see further discussion in Sections 2.2, 5 and 6). The length scales $d_{0}$ and $z_{0}$ are strongly dependent on the type of building structure in the urban environment, and accordingly several methods have been proposed to relate $d_{0}$ and $z_{0}$ to the surface morphometry. These methods are briefly summarized below. In general, a variety of methods is presently used to estimate the profiles parameters $z_{0}, u_{*}$ and $d_{0}$, and different approaches are tested later in the present paper. In order to distinguish between the different methods, additional indices are introduced for $z_{0}, u_{*}$ and $d_{0}$. An overview of the estimation methods and corresponding symbols is given in Table $\mathrm{I}$.

\subsubsection{Morphometric Methods to Determine Wind Profile Parameters}

Based on experimental studies, roughness classifications with typical values for $z_{0}$ and $d_{0}$ have been presented (Plate, 1995), and a number of methods have been proposed to determine $z_{0}$ and $d_{0}$ based on information about the surface morphometry. Grimmond and Oke (1999) presented an overview of the different methods and tested them against datasets for 11 sites in seven North American cities. The morphometric methods can be classified into three categories:

1. Height-based approaches, where $z_{0}$ and $d_{0}$ are simply expressed as fractions ( $z_{0}=f_{0} \bar{H}$ and $d_{0}=f_{d} \bar{H}$ ) of the average building height $\bar{H}$. As a rule-ofthumb, the values $f_{0}=0.1$ and $f_{d}=0.7$ are often used (Grimmond and Oke, 1999).

2. Methods that use average building height and plan areal fraction $\lambda_{p}=A_{p} / A_{T}$, where $A_{p}$ corresponds to the average plan area of roughness elements and $A_{T}$ to the total surface area. These methods take into account the variation of $z_{0}$ and $d_{0}$ with increasing building density and provide more realistic estimates than purely height dependent approaches. On the other hand, several methods in this category are not applicable over the whole range of typical urban surface characteristics and require additional input parameters that are often unknown.

3. Methods that consider average building height and frontal areal index $\lambda_{F}=$ $A_{F} / A_{T}$, where $A_{F}$ corresponds to the average frontal area of roughness elements perpendicular to the wind direction. Similar to methods of category (2), these approaches are often not easily applied due to increased input requirements.

Based on quasi-physical reasoning, Grimmond and Oke (1999) suggested curves and reasonable envelopes for the variation of $z_{0}$ and $d_{0}$ with increasing building packing density, performed sensitivity tests for the different methods and checked their conformity with these envelopes. Based on these sensitivity tests and comparisons between morphometric $z_{0}$ and $d_{0}$ estimates with wind-based $z_{0}$ and $d_{0}$ values for measurements at 11 North American sites, deficiencies of particular methods could be identified. However, it was not possible to recommend one method that performed significantly better than the others. 
TABLE I

Overview of estimation methods for the mean wind profile parameters: friction velocity, $u_{*}$, roughness length, $z_{0}$, and displacement height, $d_{0}$.

\begin{tabular}{|c|c|c|c|}
\hline Parameter & Used data & Method of estimation & Symbol \\
\hline \multirow[t]{7}{*}{ Friction velocity, $u_{*}$} & Mean wind profiles & $\begin{array}{l}\text { Least-square fits of wind profile data } \\
\text { against Equation (1), with } d_{0 m} \text { values as } \\
\text { input parameters for the displacement } \\
\text { height. The regression coefficients } \\
\text { yield then } u_{*} \text { and } z_{0}\end{array}$ & $u_{* p}$ \\
\hline & $\begin{array}{l}\text { Mean wind velocity } \\
\text { measured at a } \\
\text { reference height }\end{array}$ & $\begin{array}{l}\text { Roughness length, } z_{0} \text {, and } \\
\text { displacement height, } d_{0} \text {, are estimated } \\
\text { by a morphometric method, and } u_{*} \text { is } \\
\text { calculated by Equation (17) }\end{array}$ & $u_{* r}$ \\
\hline & \multirow[t]{5}{*}{$\begin{array}{l}\text { Turbulent shear- } \\
\text { stress profiles }\end{array}$} & Measured peak value & $u_{f m}$ \\
\hline & & Value measured above the RSL & $u_{f *}$ \\
\hline & & Local, height-dependent value & $u_{f l}$ \\
\hline & & $\begin{array}{l}\text { Peak value of the parameterized } \\
\text { profile }\end{array}$ & $u_{s}$ \\
\hline & & $\begin{array}{l}\text { Local height-dependent value of the } \\
\text { parameterized profile }\end{array}$ & $u_{s l}$ \\
\hline \multirow[t]{3}{*}{ Roughness length, $z_{0}$} & Mean wind profiles & $\begin{array}{l}\text { Least-square fits of wind profile data } \\
\text { against Equation (1), with } d_{0 m} \text { values as } \\
\text { input parameters for the displacement } \\
\text { height. The regression coefficients } \\
\text { yield then } u_{*} \text { and } z_{0}\end{array}$ & $z_{0 p}$ \\
\hline & Mean wind profiles & $\begin{array}{l}\text { Average relation between } z_{0} \text { and } \\
z_{s}-d_{S} \text { (Equation (14)), which was } \\
\text { determined based on log-law fits }\end{array}$ & $z_{0 i}$ \\
\hline & $\begin{array}{l}\text { Information about } \\
\text { building morphometry }\end{array}$ & $\begin{array}{l}\text { Morphometric method proposed in } \\
\text { present paper (Equation (16)) }\end{array}$ & $z_{0 n}$ \\
\hline \multirow[t]{3}{*}{ Displacement height, $d_{0}$} & $\begin{array}{l}\text { Shear-stress } \\
\text { parameterization }\end{array}$ & $\begin{array}{l}\text { Integral condition (Equation (4)) after } \\
\text { Jackson (1981) }\end{array}$ & $d_{0 i}$ \\
\hline & $\begin{array}{l}\text { Information about } \\
\text { building morphometry }\end{array}$ & $\begin{array}{l}\text { Morphometric method (Equation (2)) } \\
\text { proposed by MacDonald et al. (1998) }\end{array}$ & $d_{0 m}$ \\
\hline & & $\begin{array}{l}\text { Morphometric method proposed in } \\
\text { present paper (Equation (15)) }\end{array}$ & $d_{0 n}$ \\
\hline
\end{tabular}


For the analysis of the mean wind profiles measured in the present study, a morphometric method is used to estimate the displacement height $d_{0}$, which was proposed by MacDonald et al. (1998):

$$
M A_{d}: \frac{d_{0}}{\bar{H}}=1+\alpha^{-\lambda_{p}}\left(\lambda_{p}-1\right) .
$$

For the empirical parameter $\alpha$ Macdonald et al. (1998) determined $\alpha=4.43$ based on wind-tunnel data for staggered arrays of cubes. This particular method is selected, since the analysis of Grimmond and Oke (1999) has shown that the predicted $d_{0}$ values are reasonable. Furthermore, it has the advantage that it is applicable over the full range of building densities and requires only readily available input data.

\subsubsection{Integral Condition for the Displacement Height}

Jackson (1981) described the displacement height, $d_{0}$, of the mean wind profile as the level of mean momentum absorption and proposed an integral condition to determine $d_{0}$, which can be formulated as:

$$
\left(d_{0}-d_{s}\right) \overline{u^{\prime} w_{s}^{\prime}}=\int_{d_{s}}^{z_{s}}\left(\overline{u^{\prime} w_{s}^{\prime}}-\overline{u^{\prime} w_{s}^{\prime}}(z)\right) \mathrm{d} z .
$$

The quantity $\overline{u^{\prime} w_{s}^{\prime}}$ describes the peak of the shear stress profile, $\overline{u^{\prime} w^{\prime}}(z)$, and an illustration of the integral condition is presented in Figure 1. The upper sketch refers to a situation with a relatively low building density where shear stress inside the RSL increases from zero near the ground up to a peak value that is observed above roof level. With increasing building density a transition from wake-interference flow to skimming flow can be expected and inside the canopy a zone with negligible shear stress values will develop (see lower sketch of Figure 1). Grimmond and Oke (1999) determined the value $\lambda_{p} \approx 0.35$ as the lower limit for skimming-flow conditions based on $\lambda_{s}$ values of Hussain and Lee (1980). This limit is often exceeded in urban settings, particularly for street-canyon configurations. To account for the flow region inside the canopy with practically zero momentum transport, a shear-stress displacement height, $d_{s}$, is introduced in Equation (3). The mean-wind displacement height, $d_{0}$, corresponds to the level at which the grey shaded areas (Figure 1) match. The value of $d_{s}$ is correlated to the building density and negligible in areas with low building densities. Jackson (1981) interprets $d_{s}$ as the average elevation $\bar{h}$ of the surface, which can be estimated by $d_{s}=\bar{h}=(\overline{W H}) /(\bar{W}+\bar{S})$ for a two-dimensional building configuration or $d_{s}=\bar{h}=\left(A_{p} / A_{T}\right) \bar{H}=\lambda_{p} \bar{H}$ for a three-dimensional building configuration. The parameters $W$ and $\bar{H}$ correspond to the average width and height of the buildings, and $\bar{S}$ to the average street width (see also Figure 1). 

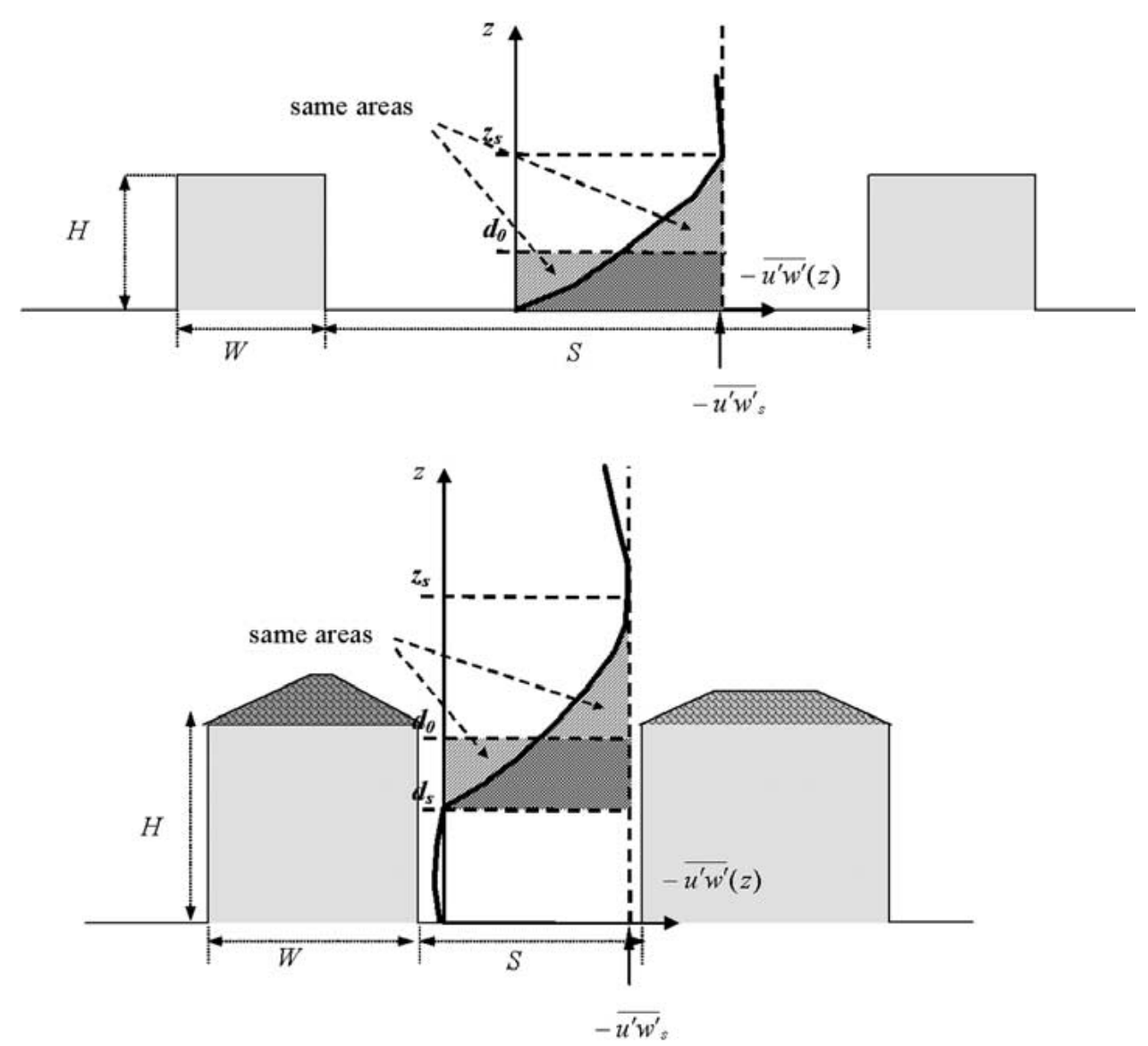

Figure 1. Determination of displacement height, $d_{0}$, according to the integral condition proposed by Jackson (1981). The upper sketch shows the application to a situation with low building density, the lower one to an urban street canyon configuration with negligible momentum flux inside the canopy.

\subsection{TURBULENCE STRUCTURE WITHIN THE ROUGHNESS SUblayeR}

Summarizing the experimental results discussed in the introduction, it can be concluded that shear stress is essentially non-constant within the RSL. A characteristic for all investigated urban landscapes is that shear stress starts to increase in the upper part of the canopy from practically zero until a peak value is reached at some distance above average roof level. Accordingly, Rotach (2001) presented a conceptual sketch for the shear-stress profile in the UBL that significantly deviates from the constant flux-layer concept. He proposed a parameterization for a local, height-dependent scaling velocity, $u_{f l}(Z)=\sqrt{-\overline{u^{\prime} w^{\prime}}(Z)}$, inside the RSL, in which the length scale, $Z$, was based on the height, $z_{f m}$, at which the shear-stress peak 
was measured, and the velocity scale related to the magnitude of the shear-stress peak, $u_{f m}=\sqrt{-\overline{u^{\prime} w_{\max }^{\prime}}}$ :

$$
\left(\frac{u_{f l}(Z)}{u_{f m}}\right)^{b}=\sin \left(\frac{\pi}{2} Z\right)^{a}
$$

for $Z \leq 1$.

In the definition of the non-dimensional height, $Z=z^{\prime} / z_{f m}^{\prime}=\left(z-d_{0}\right) /\left(z_{f m}-\right.$ $d_{0}$ ), the displacement height, $d_{0}$, of the mean wind profile is used as the reference level. Rotach (2001) associated $d_{0}$ with the level of zero stress, which does not correspond to the definition of $d_{0}$ according to Jackson (1981). The parameterized profile (Equation (4)) was fitted against full-scale data, and the empirical constants yielded $a=1.28$ and $b=3.0$. Martilli et al. (2002) found a good agreement between Equation (4) and their results from numerical simulations with a mesoscale model using urban surface exchange parameterizations.

As mentioned in the introduction, Rotach (2001) associated the depth of the urban RSL, $z_{*}$, with $z_{f m}$ and interpreted $u_{f m}$ as the relevant velocity scale for the urban ISL, i.e. the friction velocity was estimated as $u_{*}=u_{f m}$. In other studies, shear-stress peak values were considered as indications for local flow disturbances (Oikawa and Meng, 1995; Louka, 1999), and it was concluded that $z_{*}>z_{f m}$. Furthermore, it was assumed that for $z \geq z_{*}$, a constant flux layer with $\left|\overline{u^{\prime} w^{\prime}}(z)\right|<$ $u_{f m}^{2}$, exists. The friction velocity was estimated from $u_{*}=u_{f^{*}} \equiv \sqrt{-\overline{u^{\prime} w^{\prime}}\left(z_{*}\right)}$ and, consequently, smaller than $u_{f m}$. Cheng and Castro (2002) conclude, from their wind-tunnel study of flow over rough surfaces, that shear stress and mean flow data solely from the ISL do not allow determination of appropriate surface parameters. Therefore, one of the main objectives of our wind-tunnel study will be to clarify the definition of $u_{*}$ in urban areas.

\section{Experimental Set-Up}

A detailed 1:200 scale model of the building structure in the central part of Nantes, France was constructed and investigated in a neutral boundary-layer wind tunnel at the University of Karlsruhe (NATUK). The boundary layer in the approach flow was formed by vortex generators at the entrance and $20 \mathrm{~mm}$ high Lego roughness elements mounted on the wind-tunnel floor. More information regarding technical details of NATUK and the mean flow and turbulence characteristics realized in NATUK are given in Kastner-Klein (1999). The wind-tunnel model covered a realworld region of about $400 \mathrm{~m}$ in diameter. Rue de Strasbourg (RdS), a relatively long street with high buildings on both sides, was selected as the main area of investigation and placed in the centre of the model. This choice was motivated by full-scale observations conducted in this street (Vachon et al., 1999, 2001). A city map of the area reconstructed in the wind-tunnel model is shown in Figure 


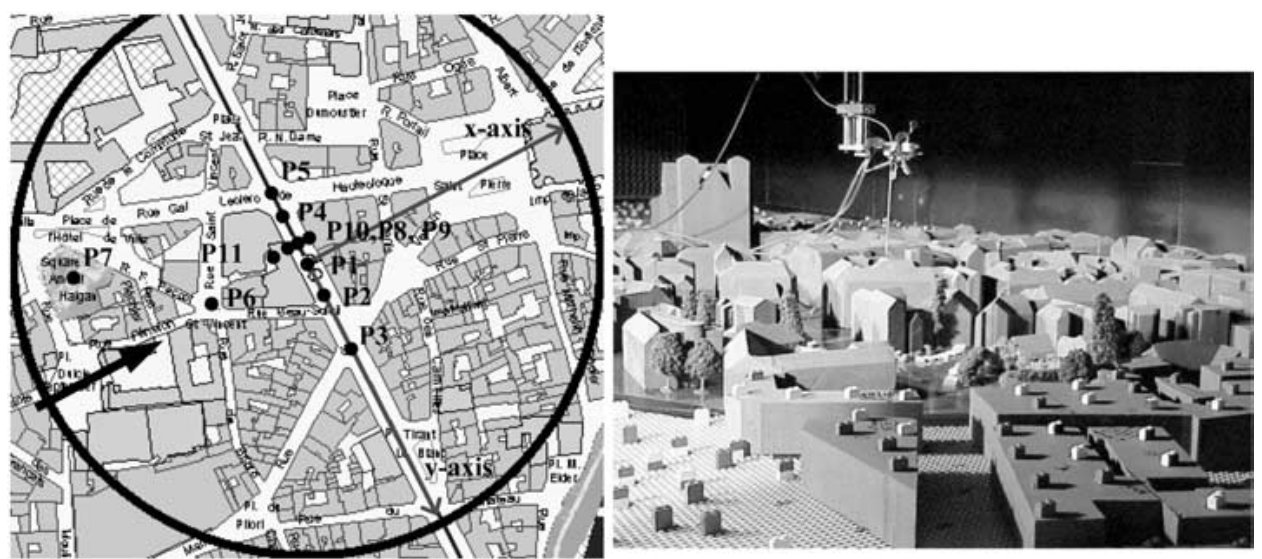

Figure 2. Area in the centre of Nantes reconstructed in the wind-tunnel model (circle) with the location of profiles (left) and photograph of the model installed in the boundary-layer wind tunnel (right).

2 together with a photograph of the model installed in the wind tunnel. It gives an impression of the complexity of the urban landscape in the investigated area. The obstacles seen in the foreground correspond to additional, idealized buildings, which were modelled taking into account the average building height and density in this area in order to increase the length of the urban fetch. In total, the fetch of urban type buildings, up to the centre of the model, was about $1.30 \mathrm{~m}$ in the wind tunnel.

Vertical velocity profiles were measured with a Laser Doppler velocimeter (details about the measurement technique are given in Kastner-Klein, 1999) at the positions P1 to P11 marked in Figure 2 and upstream of the model (position REF). The latter profiles deliver information about the approach flow conditions. At each location, two vertical profiles (sampling frequency $20 \mathrm{~Hz}$ and sampling time $102 \mathrm{~s}$ ) with simultaneously sampled time series of two velocity components ( $u$ plus $w$; and $u$ plus $v$ ) were taken with a different probe orientation. The velocity components are defined in the following way: $u$ parallel to x-axis, $v$ parallel to yaxis (see Figure 2) and $w$ the vertical component. The profile locations P1 to P11 were selected in order to trace the boundary-layer development over the modelled urban area and to investigate the horizontal flow variability inside and above the $\mathrm{RdS}$ street canyon, which was oriented perpendicular to the wind direction. The coordinates of the positions are given in Table II along with the symbols used for each profile in the following diagrams. The displacement height, $d_{0 m}$, roughness length, $z_{0 p}$, and friction velocity, $u_{* p}$, also given in Table II, are further discussed in the next paragraph.

The distribution of building heights in the area around RdS, which was reconstructed in the wind-tunnel model, is shown in Figure 3. The height values, indicated by different colours, refer to the level of the model-building roof edges. 
TABLE II

Description of sampling point locations and corresponding wind-profile parameters determined by a morphometric method $\left(d_{0 m}\right)$ and fitting the measured mean wind profiles against the $\log \operatorname{law}\left(z_{0 p}, u_{* p}\right)$.

\begin{tabular}{|c|c|c|c|c|c|c|}
\hline & $x(\mathrm{~m})$ & $y(\mathrm{~m})$ & $d_{0 m}(\mathrm{~mm})$ & $z_{0 p}(\mathrm{~mm})$ & $u_{* p}\left(\mathrm{~m} \mathrm{~s}^{-1}\right)$ & Symbol \\
\hline P1 & 0.0 & 0.0 & 96.00 & 6.87 & 0.70 & th \\
\hline $\mathrm{P} 2$ & 0.0 & 0.12 & 88.00 & 5.18 & 0.66 & $x$ \\
\hline P3 & 0.0 & 0.28 & 82.00 & 3.09 & 0.58 & $x$ \\
\hline P4 & 0.0 & -0.14 & 99.00 & 6.69 & 0.69 & 0 \\
\hline P5 & 0.0 & -0.23 & 87.00 & 15.36 & 0.91 & $\bigcirc$ \\
\hline P6 & -0.33 & 0.0 & 90.00 & 6.10 & 0.67 & $\vec{z}$ \\
\hline P7 & -0.66 & -0.23 & 75.00 & 4.48 & 0.64 & $\star$ \\
\hline P8 & 0.0 & -0.7 & 101.00 & 5.19 & 0.65 & $\diamond$ \\
\hline P9 & 0.02 & -0.07 & 101.00 & 5.29 & 0.65 & $\nabla$ \\
\hline P10 & -0.02 & -0.07 & 101.00 & 5.30 & 0.65 & $\triangle$ \\
\hline P11 & -0.08 & -0.07 & 101.00 & 3.99 & 0.61 & $\Delta$ \\
\hline REF & -1.80 & 0.0 & 0.2 & 0.86 & 0.42 & solid line \\
\hline
\end{tabular}

The point of origin corresponds to the location of sampling position P1. It becomes obvious that the building-density distribution in the upwind area is rather asymmetric. In the area to the right of $\mathrm{P} 1(y>0)$ the packing density is rather high. In this region, only a few narrow streets separate dense building clusters with almost completely covered backyard zones. On the other hand, open spaces and smaller, less dense building clusters characterize the area to the left of $\mathrm{P} 1(y<0)$.

The differences in packing density are also well seen in Figure 4 in which the average building height, $\bar{H}$, and plan areal fraction, $\lambda_{p}$, along RdS are plotted. Using the building database reflected in Figure 3 , the $\bar{H}$ and $\lambda_{p}$ values are calculated for each grid point along the centre line of RdS by taking into account the geometry data in an upwind elliptical footprint area. The major radii of the ellipses are set to $a=0.4 \mathrm{~m}$ (filled symbols) and $a=0.2 \mathrm{~m}$ (open symbols) and the minor radii are equal to $b=(2 / 3) a$. The variation of $a$ is considered, in order to trace the alongwind homogeneity of the upwind building structure. In Section 4 , the $\bar{H}$ and $\lambda_{p}$ values are used to determine the displacement height, $d_{0}$, of the mean wind profile with a morphometric method (Equation (2)), and the sensitivity of the $d_{0}$ estimates to the size of the upwind footprint area is tested.

When analyzing the wind-tunnel data, the growth of an internal boundary layer (IBL) developing over the modelled urban area must be taken into account. Theories and experimental results regarding the IBL depth and flow profiles inside the IBL are reviewed in Garratt (1990) and Kaimal and Finnigan (1994). For the urban area modelled in the wind tunnel, a rough estimate of the roughness length 


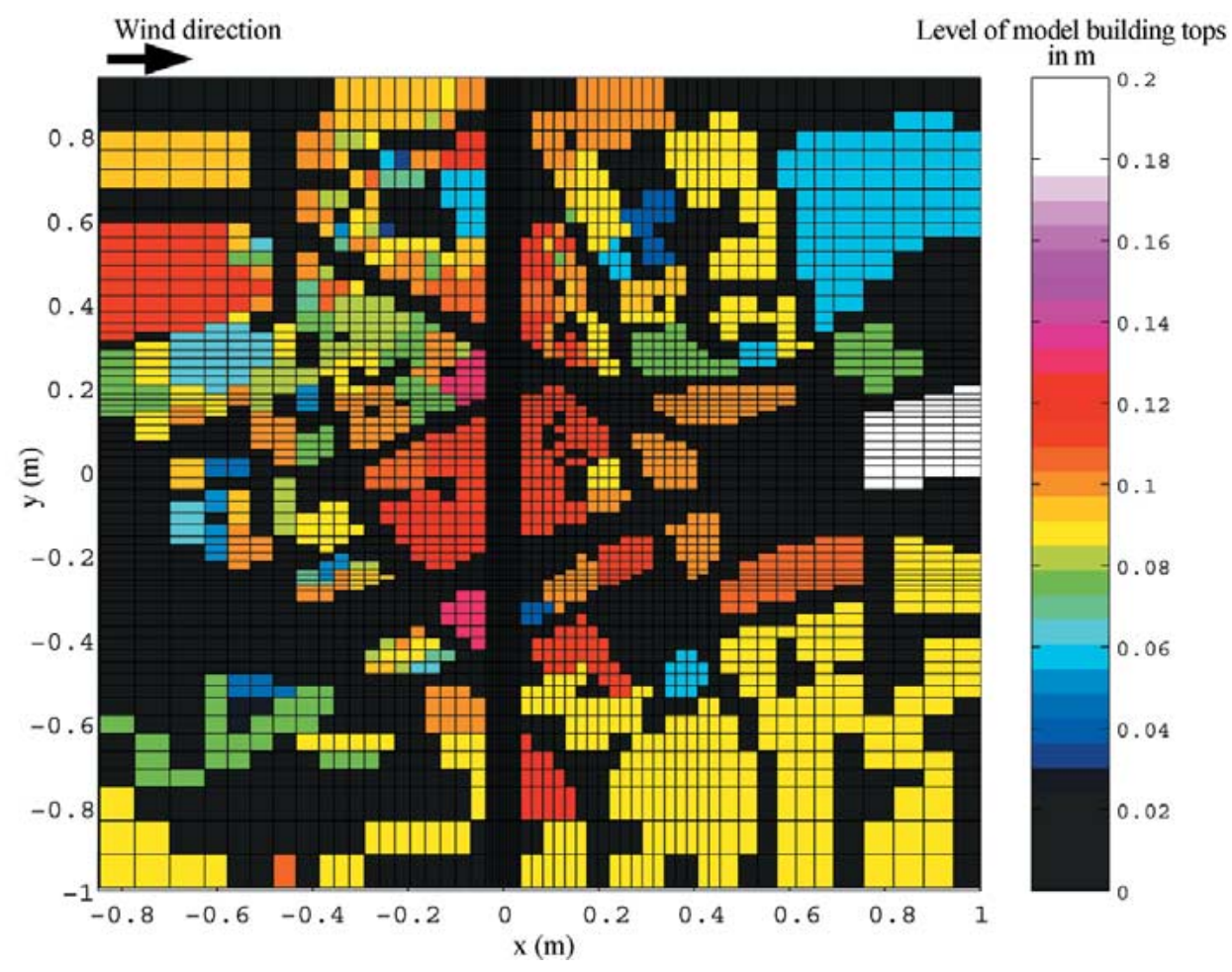

Figure 3. Distribution of building heights (level of roof edges in $\mathrm{m}$ ) in the wind-tunnel model of the central part of Nantes, France. Rue de Strasbourg can easily be located as the dark vertical stripe at $x \approx 0$.

$\left(z_{02} \approx 10 \mathrm{~mm}\right.$ ) can be made using $0.1 \mathrm{~m}$ as the value of the average building height and the empirical relation $z_{0} \approx 0.1 \bar{H}$ given in Grimmond and Oke (1999). The roughness length in the approach flow section, $z_{01}$, is known to be approximately $1 \mathrm{~mm}$. Based on these $z_{0}$ values, the IBL growth along the urban model can be calculated using formulae proposed by Elliot (1958) and Panofsky and Dutton (1984). According to these estimates, the IBL extends up to the highest measurement level $(0.35 \mathrm{~m})$ in the main area of investigation (RdS), which is encircled by an upwind urban fetch of about $1.30 \mathrm{~m}$, whereas influences of the roughness change should be visible in the flow profiles at the positions P6 and P7, which are located further upwind (at P7 the upwind urban fetch is only about $0.5 \mathrm{~m}$ ).

\section{Experimental Results}

The mean wind velocity, turbulent shear stress and turbulent kinetic profiles obtained in the Nantes wind-tunnel study are plotted in Figure 5 (see Table II for a list of symbols). As a reference length, an average value of the building height, $H_{r}$, in the area of investigation is used. This average building height is estimated 


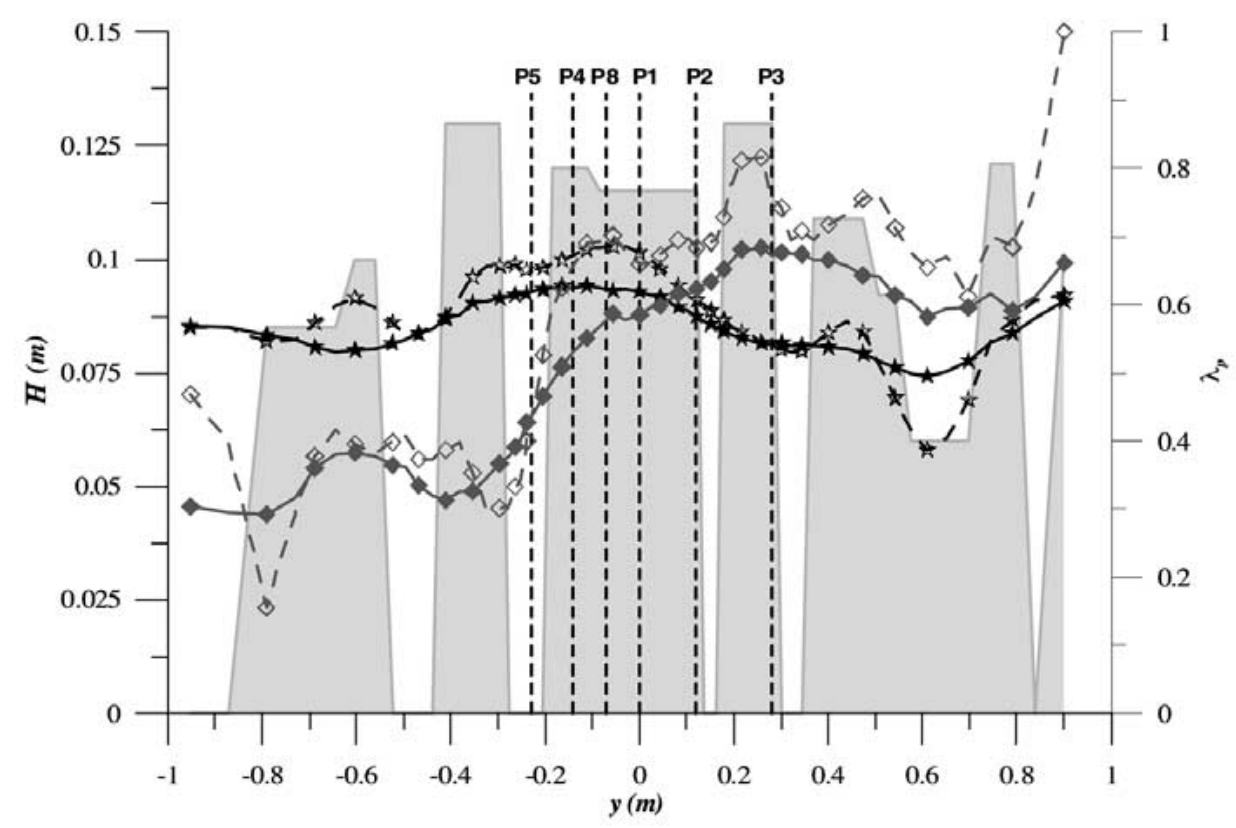

Figure 4. Distribution of average building heights, $\bar{H}$, (stars) and plan areal fraction, $\lambda_{p}$, (diamonds) along RdS (the dashed vertical lines indicate the profile locations in $\mathrm{RdS}$ ). The calculations are based on the building structure in an upwind elliptic footprint area with the major radii $a=0.4 \mathrm{~m}$ (filled symbols) and $a=0.2 \mathrm{~m}$ (open symbols), where in both cases, the minor radii equal $b=(2 / 3) a$. The grey shaded area illustrates the level of building tops at the upwind side of RdS.

to be $0.1 \mathrm{~m}$, but the roof level of single buildings flanking RdS is up to $0.13 \mathrm{~m}$ above street level (see grey shaded area in Figure 4). The influence of building pattern irregularities on the mean flow, shear stress, and turbulence kinetic energy distributions are clearly noticeable up to the level $z \approx 3 H_{r}$. Above this level, the variation of the mean velocity profiles is less than $2 \%$, the variation of the turbulence kinetic energy profiles less than $10 \%$ and the variation of the shear stress profiles approximately $15 \%$.

The observed wind profiles can be classified in two types. The first type includes characteristic canyon-flow profiles with almost zero or even negative mean wind velocities inside the canopy (black curves in Figure 5). Profiles referring to the second type are representative of the wind regime around intersections or in open squares and characterized by higher mean and turbulent flow velocities in the canopy layer (grey curves in Figure 5). Shear stress and turbulence kinetic energy profiles corresponding to both flow types in many cases show pronounced maxima in the region just above the average roof level (between $H_{r}$ and $1.5 H_{r}$ ), which is very similar to the results obtained in a recent full-scale study (Christen et al., 2002). A distinct constant-flux layer could not be observed. In Section 3, it was estimated that the IBL extends up to $z \approx 0.3-0.35 \mathrm{~m}$, i.e. the highest measurement level, in the main area of investigation. However, it is typically assumed (Kaimal 
(a)

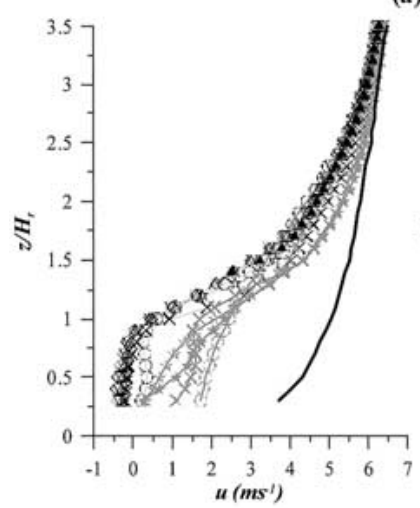

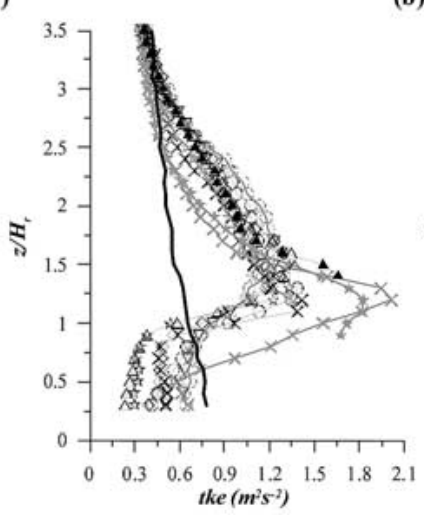

(b)

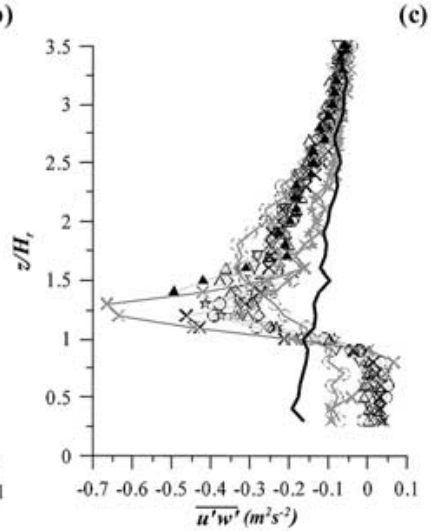

Figure 5. Measured profiles of (a) the mean $u$ component, (b) turbulence kinetic energy and (c) turbulent shear stress of the Nantes wind-tunnel study (symbols according to Table II).

and Finnigan, 1994) that the region, in which a constant-stress layer (or ISL) in equilibrium with the new surface is achieved, corresponds only to the lowest $10 \%$ of the IBL. Accordingly, the equilibrium layer covers only a few tens of $\mathrm{mm}$ in the region of RdS. This is certainly related to the fact that only a rather limited urban area could be reconstructed in the wind tunnel. On the other hand, changes in surface morphology are fairly typical in urban areas and it is still an open question if distinct constant-stress layers exist at urban sites. This was also one of the conclusions of Cheng and Castro (2002), who used fairly extensive fetches in their wind-tunnel studies with idealistic urban surfaces. Furthermore, Rotach (1999) argued that the RSL can amount to several tens of metres in urban areas, and so span across the lowest $10 \%$ of the atmospheric boundary layer such that no ISL is present.

To cope with the problem of horizontal inhomogeneity of the flow in the RSL, it is recommended to analyze spatially averaged profiles (Raupach, 1980; MacDonald, 2000; Cheng and Castro 2002). However, the significance of spatially averaged profiles is questionable for urban street-canyon configurations, at least if they are characterized by skimming flow regimes. For loosely-packed, regularly-spaced obstacle arrays a spatially averaged mean wind profile is of practical relevance and can be considered descriptive of the mean advection of pollutants released in the RSL. At urban sites with skimming-flow characteristics, horizontal advection is highly variable and depends on the particular location and on the wind direction. Pollutants released near intersections may be advected along a particular street, and channelling of the plume can be expected. In long street canyons, pollutants can be trapped and for certain wind directions horizontal advection inside the UCL becomes negligible, and vertical mixing becomes a dominant dispersion mechanism. We, therefore, argue that a spatially averaged profile will not deliver representative 
information about the flow regime in RdS, and consequently aimed our analysis at the description of the individual profiles.

Despite the problem of the rather shallow ISL above the modelled urban landscape, the measured mean wind profiles were approximated by the log law (Equation (1)). We realize that this approach lacks a firm theoretical background, but decided to check to what extent a semi-logarithmic relationship expressed by Equation (1) describes the measured profiles. For this purpose, $d_{0}$ is estimated for each sampling location using Equation (2), and using the $\bar{H}$ and $\lambda_{p}$ values presented in Figure 4. The $M A_{d}$ values (Equation (2)) for the displacement height, in the following referred to as $d_{0 m}$, have been used as input parameters for regressions to the upper part $\left(2.5 d_{0 m} \leq z \leq 3.5 d_{0 m}\right)$ of the velocity profiles according to Equation (1). The regression coefficients then yield the roughness length $z_{0 p}$ and friction velocity $u_{* p}$ (where the index $p$ stands for the described profile fitting procedure). By using $d_{0 m}$ as input information when fitting the wind profile, the problem that three unknown parameters appear in Equation (1) is overcome, given that the regression only allows determination of two parameters.

The $d_{0 m} / \bar{H}$ ratios (diamonds) for each sampling location are presented in Figure $6 \mathrm{a}$ as a function of plan area index $\lambda_{p}$. The open symbols refer to results obtained based on $\bar{H}$ and $\lambda_{p}$ values estimated for an upwind footprint area with $a=0.2 \mathrm{~m}$, while the closed, grey symbols show the results obtained if an upwind area with $a=0.4 \mathrm{~m}$ is considered. The solid line and grey shaded area plotted in Figure 6 indicate the curve and reasonable envelopes for $d_{0} / \bar{H}$ and $z_{0} / \bar{H}$ suggested by Grimmond and Oke (1999). The grey lines in Figure 6 will be discussed in Section 6 . The larger upwind footprint area $(a=0.4 \mathrm{~m})$ results in lower $d_{0 m} / \bar{H}$ values than the smaller upwind footprint area $(a=0.2 \mathrm{~m})$. As shown in Figure $6 \mathrm{~b}$, the values for the roughness length, $z_{0 p}$, are consequently higher when the $d_{0 m}$ estimate is based on the larger footprint area (closed symbols). Overall, the $d_{0 m}, z_{0 m}$ and $u_{* p}$ values appear to be somewhat more realistic if the smaller footprint area is employed. The corresponding values are given in Table II.

A comparison of the mean wind profiles, normalized using $d_{0 m}, z_{0 p}$, and $u_{* p}$ with the log law (dashed line) is presented in Figure 7a. It becomes obvious that the measured profiles agree quite well with a logarithmic profile even though a distinct constant stress layer does not exist. For the height range $z \geq 2 d_{0 m}$ the deviation between the fitted log profile and the measured data is less than $\pm 5 \%$ (see Figure $7 \mathrm{~b}$ ). Thus, it can be concluded that the profile parameters $z_{0 p}, u_{* p}$ and $d_{0 m}$ are appropriately estimated and that the wind profiles in the developing UBL above $z \approx 2 d_{0}$ are well approximated by a logarithmic wind profile.

Although this result is rather promising, the determination of the profile parameters in urban areas still remains problematic. The fact that a set of parameters can be found, which describes measured profiles accurately enough, is not particularly useful for practical applications, when usually only a few mean wind velocity measurements are available. The discussion in the introduction has for 
(a)
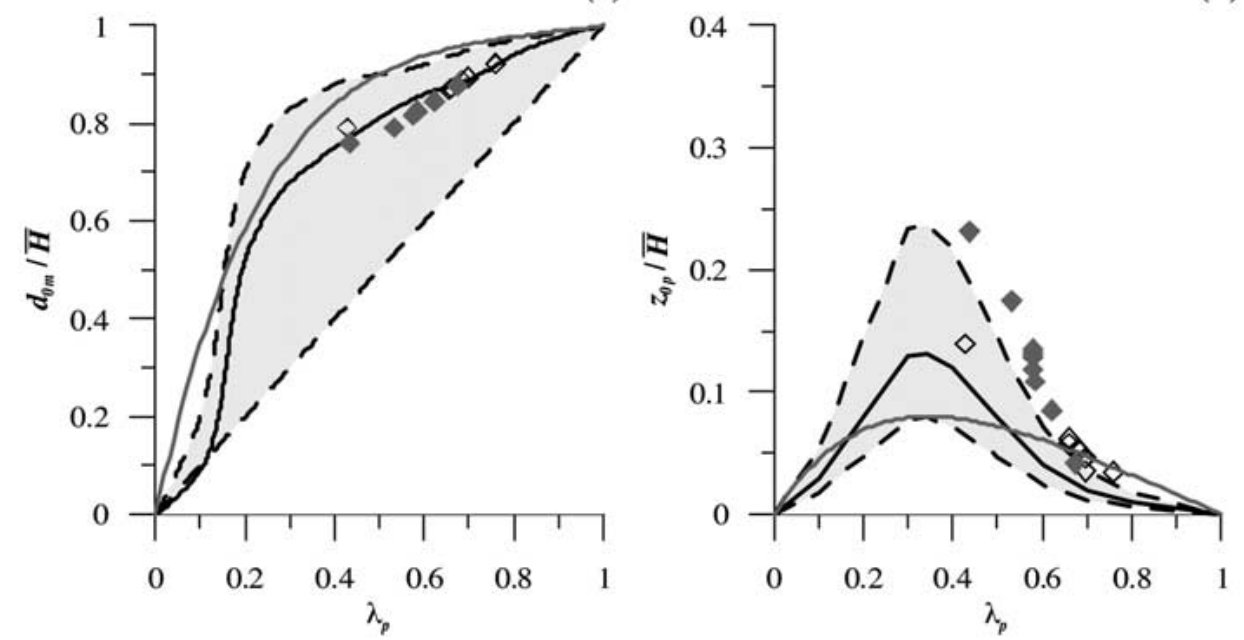

(b)

Figure 6. (a) Displacement height, $d_{0 m}$, as a function of plan area index, $\lambda_{p}$, and (b) corresponding values for the roughness length, $z_{0 p}$, determined by fitting the log law (Equation (1)) to the measured mean wind profiles. The grey shaded area delimited by the dashed lines and the characterizing dark full line denote the physically reasonable range as suggested by Grimmond and Oke (1999). The grey lines are introduced in Section 6. Diamonds indicate the calculated values at the 11 sites in the Nantes study, filled for $a=0.4 \mathrm{~m}$, open for $a=0.2 \mathrm{~m}$ (see text for details).

(a)

(b)
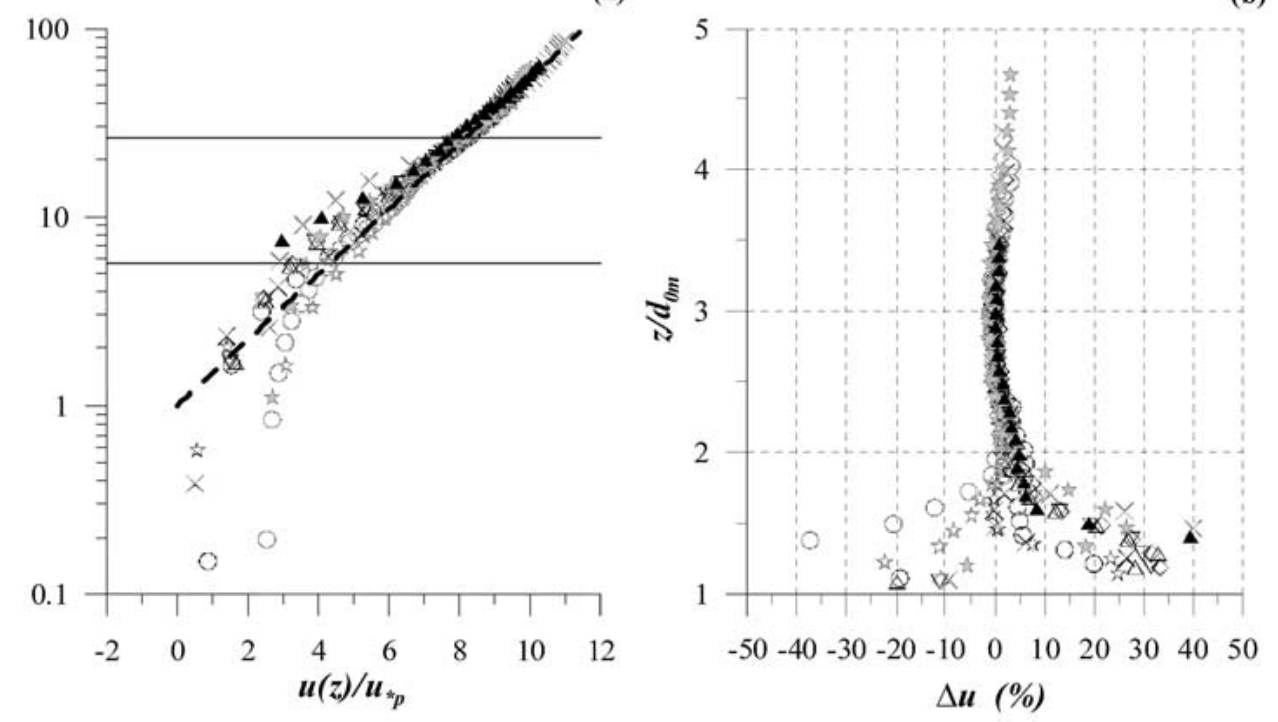

Figure 7. (a) Fitting of mean wind profiles against the log law and (b) deviation between fitted log-profile and measured data $\Delta u=\left(u_{\mathrm{fit}}-u_{\mathrm{obs}}\right) / u_{\mathrm{obs}}$ in percent. See Table II for values of the profile parameters $d_{0 m}, z_{0 p}, u_{* p}$ and a list of symbols. The dashed line in (a) denotes Equation (1), and the horizontal lines indicate the range of $\left(z-d_{0 m}\right) / z_{0 p}$ values corresponding to $z=2 d_{0 m}$ for the various profiles. 
instance shown that $u_{*}$ is not clearly defined even when shear-stress profile data are available.

In order to clarify some of the questions related to the definition of $u_{*}$ in urban areas, the correlation between the values of the friction velocity $u_{* p}$ and velocity scales determined from the measured shear stress profiles has been analyzed. In previous studies (see discussion in Sections 1 and 2.2), the friction velocity $u_{*}$ was estimated based on the shear-stress peak value (Rotach, 2001) or a shear-stress measurement above the RSL (Oikawa and Meng, 1995). For the Nantes study, the level $z=3 H_{r}$ can be viewed as an upper limit for the RSL, since the influence of building pattern irregularities above $z=3 H_{r}$ is not very pronounced (see Figure 5 and the discussion in Section 4). In Figure 8, the $u_{* p}$ values are compared with velocity scales $u_{f m}$ based on the measured shear-stress peak values and with $u_{f *}$ based on the shear-stress values at $z=z_{*}$. In general, the magnitudes of $u_{f m}$ and $u_{* p}$ are fairly close. However, the shear-stress profiles can show pronounced peaks just above rooftop, such as at the positions P2 and P3, which resemble only local flow disturbances but not integral characteristics. At such locations, the friction velocity, $u_{* p}$, is overestimated by $u_{f m}$. On the other hand, the velocity scale $u_{f *}$ follows the tendencies of the $u_{* p}$ values, but is significantly lower in magnitude than $u_{* p}$.

These results indicate that a representative scaling velocity inside and above an urban RSL can be related to shear-stress peak values, provided the influence of local flow disturbances is taken into account. Accordingly, the conceptual framework proposed by Rotach (2001), which is summarized in Section 2.2, is applied for a further analysis of the wind-tunnel data. A method that allows for local flow disturbances, which can result in non-representative, extreme shear stress values at roof level, is presented in the following section.

\section{Shear Stress Parameterization}

All measured turbulent shear-stress profiles presented in Figure $5 \mathrm{c}$ are characterized by small absolute values inside the urban canopy, a strong increase in the upper part of the canopy and a peak value in a zone above roof-level. The height where the maximum is observed, as well as its magnitude, vary with the profile location. Provided the shear-stress profiles are self-similar, an appropriate scaling for the profiles can be expressed by:

$$
\frac{\overline{u^{\prime} w^{\prime}}}{\overline{u^{\prime} w_{s}^{\prime}}}=F\left(\frac{\hat{z}}{\hat{z}_{s}}\right) \text {. }
$$

The length scale $\hat{z}$ and turbulent flux scale $\overline{u^{\prime} w_{s}^{\prime}}$ are related to the height $z_{f m}$ where the peak value is observed and its magnitude $\overline{u^{\prime} w_{\max }^{\prime}}$, respectively. However, the measured shear-stress peak value can be strongly affected by local flow disturbances (see discussion in the previous section) and is therefore not a good choice for 


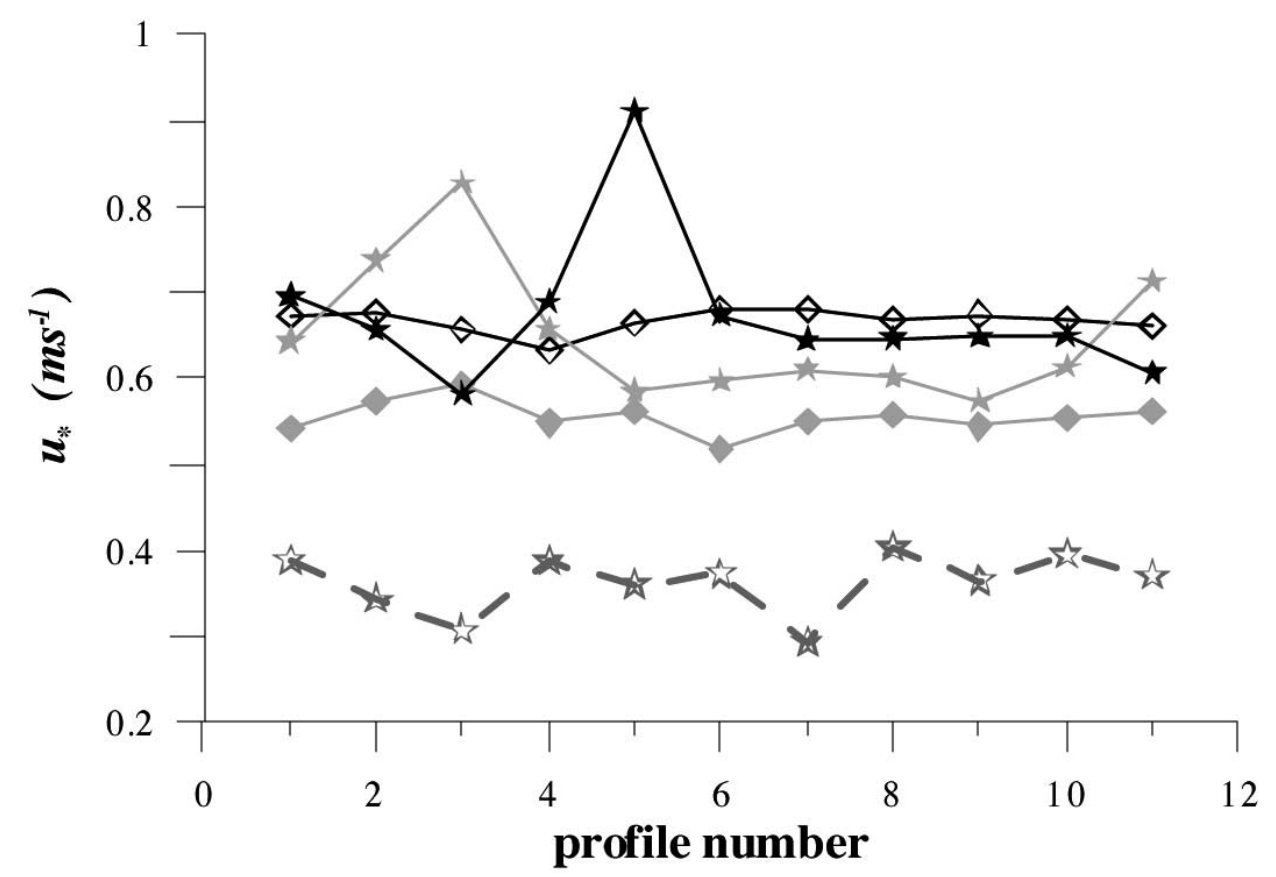

Figure 8. Comparison of different velocity scales: $u_{* p}$ (black, filled stars); $u_{f m}$ (grey, filled stars) and $u_{f *}$ (open stars) based on measured shear stress values; $u_{s}=\sqrt{-\overline{u^{\prime} w_{s}^{\prime}}}$ (grey diamonds) based on fitted shear stress peak values; $u_{* r}$ (open, black diamonds) calculated by Equation (17). For the definition and notation of the different velocity scales, see Table I. Note that some of these velocity scales will be introduced in Section 6 (see text for more details).

the scaling parameters $\hat{z}_{s}$ and $\overline{u^{\prime} w_{s}^{\prime}}$. To attenuate such locally produced, extreme peak values, the measured profiles are approximated by curve fitting (non-linear regression) and the scales $\hat{z}$ and $\overline{u^{\prime} w_{s}^{\prime}}$ are determined from the fitted curves. The fitted curves have the general form

$$
\overline{u^{\prime} w^{\prime}}(z)=a(\hat{z})^{2} \exp \{-b \hat{z}\}
$$

which captures both the increasing absolute values of $\overline{u^{\prime} w^{\prime}}$ in the upper part of the canopy and the decreasing absolute $\overline{u^{\prime} w^{\prime}}$ values at higher levels. This particular function, which is to some extent arbitrarily chosen, describes the measured results fairly well (see further analysis) and has the advantage that mathematical operations can be easily processed.

Following the ideas of Jackson (1981) (described in Section 2.1.2) a shear-stress displacement height, $d_{s}$, is used to calculate the relative height coordinate, $\hat{z}$, as follows:

$$
\hat{z}=z-d_{s} .
$$



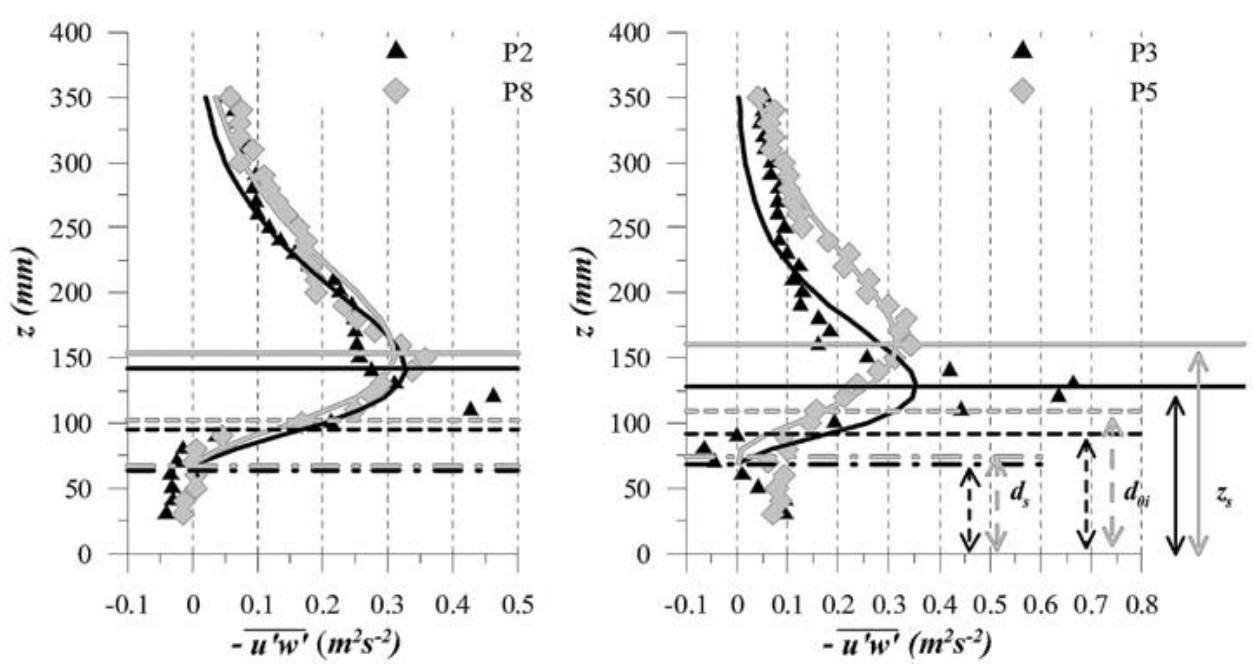

Figure 9. Illustration of the shear-stress approximation and corresponding length scales for four measured shear-stress profiles.

This displacement height accounts for the flow region inside a street canyon with almost zero momentum transport. The scaling parameters $\hat{z}_{s}$ and $\overline{u^{\prime} w_{s}^{\prime}}$ are related to the parameters $a$ and $b$ of the curve fits according to:

$$
\hat{z}_{s}=z_{s}-d_{s}=2 / b \Leftrightarrow z_{s}=d_{s}+2 / b
$$

and

$$
\overline{u^{\prime} w_{s}^{\prime}} \equiv \overline{u^{\prime} w^{\prime}}\left(z_{s}\right)=a(2 / b)^{2} \exp \{-2\} .
$$

Using the expressions (8) and (9), Equation (6) can be reformulated to:

$$
\frac{\overline{u^{\prime} w^{\prime}}}{\overline{u^{\prime} w_{s}^{\prime}}}=\left(\frac{\hat{z}}{\hat{z}_{s}}\right)^{2} \exp \left\{2\left(1-\hat{z} / \hat{z}_{s}\right)\right\}
$$

The selected curve fit follows the self-similarity criterion imposed by Equation (5). The approximation of the measured shear-stress profiles is illustrated in Figure 9 by a comparison of four measured and fitted profiles. Obviously, the fitted profiles resemble the general shapes of the measured profiles, but as intended extreme peak values are truncated. The method, by which the values of the mean-wind displacement height, $d_{0 i}$, (also included in Figure 9) are determined is discussed in Section 6.

The scaling with $\hat{z}_{s}$ and $\overline{u^{\prime} w_{s}^{\prime}}$ of all turbulent flux profiles measured in the wind tunnel is presented in Figure 10a. The profiles collapse well, which indicates the self-similarity of the shear-stress profiles and the appropriate choice of scaling parameters. The solid, black line plotted in Figure 10a corresponds to the curve 
(a)
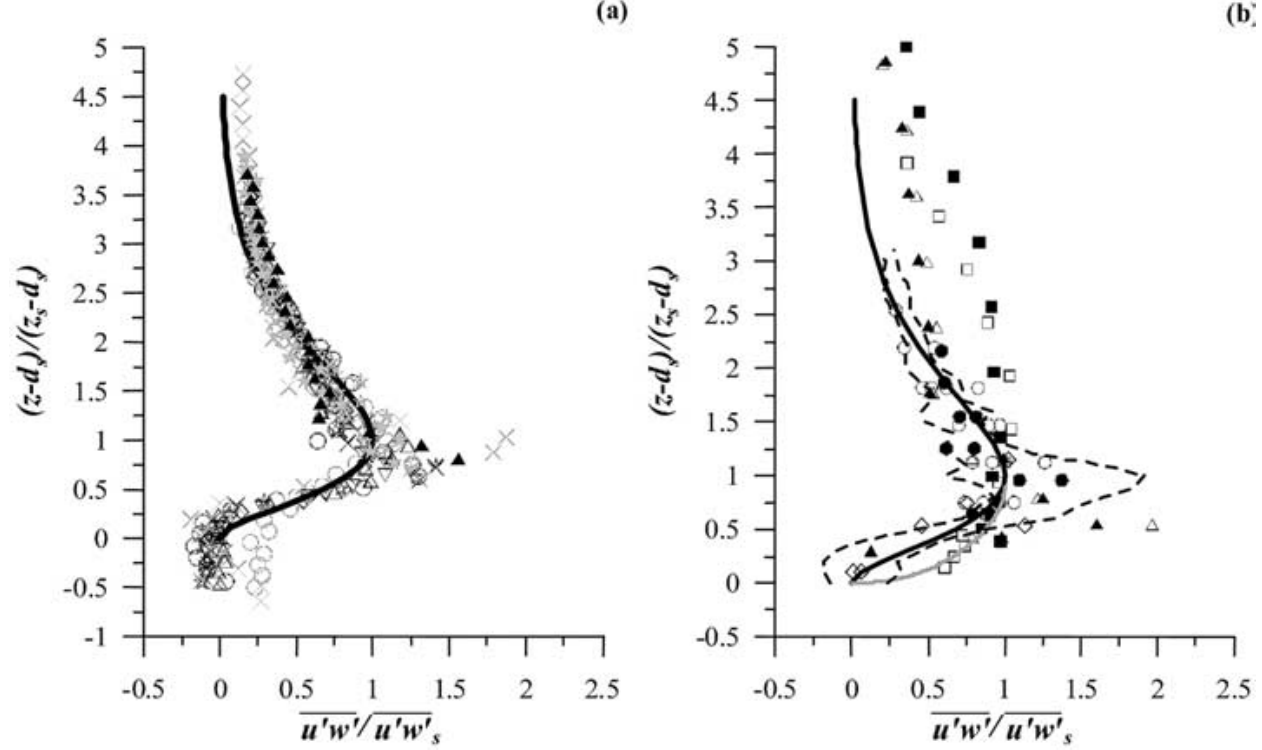

Figure 10. (a) Scaling of wind-tunnel shear stress profiles measured in the Nantes study (symbols according to Table II) and (b) comparison of proposed shear-stress parameterizations according to Equations (4) and (10) with full-scale and wind-tunnel data published in the literature (Rotach (1993a): $\diamond$; Louka (1999), smooth-rough case: $\bigcirc$, rough-rough case: ๑; Oikawa and Meng (1995):

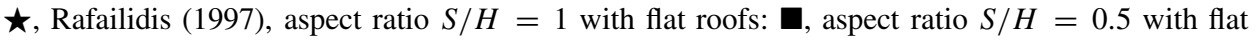
roofs: $\square$, aspect ratio $S / H=1$ with slanted roofs: $\boldsymbol{\Lambda}$, aspect ratio $S / H=0.5$ with slanted roofs: $\triangle$. Black line: Equation (10), grey line: Equation (4), dashed lines: scatter range of RdS profiles shown in (a)).

fit according to Equation (10). It agrees well with the measured profiles, except for the region near the maximum, since these peak values are intentionally attenuated.

Figure 10b shows a comparison of full-scale data and wind-tunnel data for regular street canyon arrays, along with the shear-stress parameterizations according to Equations (4) and (10) The dashed lines indicate the scatter of the RdS profiles, which are individually plotted in Figure 10a. Inside the RSL a good comparison between measurements and parameterized profiles is observed. Even the profiles for the regular arrays with slanted roofs are fairly well described by Equation (10), although they are characterized by very pronounced shear-stress peak values just above roof level. Normalization with directly measured shear-stress peak values would not be successful in this case. At higher elevations deviations between measured data and Equation (10) can be noted. However, one must take into account that the shear-stress parameterization is intended to describe the RSL characteristics rather than the characteristics of the upper part of the UBL. In order to verify parameterizations for this flow region, further full-scale and wind-tunnel studies are required. These studies should investigate the extent to which the flow above 
TABLE III

Scaling parameters for shear stress and mean velocity profiles derived on the basis of a proposed shear-stress parameterization (Equation (10)).

\begin{tabular}{|c|c|c|c|c|c|c|c|}
\hline & $\begin{array}{l}d_{S} \\
(\mathrm{~mm})\end{array}$ & $\begin{array}{l}z_{s} \\
(\mathrm{~mm})\end{array}$ & $\begin{array}{l}-\overline{u^{\prime} w_{s}^{\prime}} \\
\left(\mathrm{m}^{2} \mathrm{~s}^{-2}\right)\end{array}$ & $\begin{array}{l}u_{s}=\sqrt{-\overline{u^{\prime} w_{s}^{\prime}}} \\
\left(\mathrm{m} \mathrm{s}^{-1}\right)\end{array}$ & $\begin{array}{l}d_{0 i} \\
(\mathrm{~mm})\end{array}$ & $\begin{array}{l}z_{0 i} \\
(\mathrm{~mm})\end{array}$ & $\begin{array}{l}u_{* r} \\
\left(\mathrm{~m} \mathrm{~s}^{-1}\right)\end{array}$ \\
\hline P1 & 68 & 152 & 0.293 & 0.54 & 102 & 6.1 & 0.67 \\
\hline $\mathrm{P} 2$ & 64 & 142 & 0.327 & 0.57 & 95 & 5.6 & 0.68 \\
\hline P3 & 68 & 128 & 0.355 & 0.60 & 92 & 4.3 & 0.66 \\
\hline P4 & 67 & 151 & 0.300 & 0.55 & 100 & 6.1 & 0.63 \\
\hline P5 & 74 & 161 & 0.314 & 0.56 & 109 & 6.3 & 0.66 \\
\hline P6 & 45 & 142 & 0.269 & 0.52 & 84 & 7.0 & 0.68 \\
\hline P7 & 27 & 110 & 0.304 & 0.55 & 60 & 6.0 & 0.68 \\
\hline P8 & 67 & 154 & 0.310 & 0.56 & 102 & 6.3 & 0.67 \\
\hline P9 & 62 & 153 & 0.296 & 0.54 & 98 & 6.6 & 0.67 \\
\hline P10 & 69 & 155 & 0.307 & 0.55 & 104 & 6.2 & 0.67 \\
\hline P11 & 83 & 155 & 0.317 & 0.56 & 112 & 5.2 & 0.66 \\
\hline $\begin{array}{l}\text { mean value, } \\
\text { (P6, P7 } \\
\text { excluded) }\end{array}$ & & & & 0.56 & 102 & 5.9 & 0.67 \\
\hline
\end{tabular}

realistic urban landscapes reaches an equilibrium, in which a constant flux layer is present.

The values of the scaling parameters $d_{z}, z_{s}$ and $\overline{u^{\prime} w_{s}^{\prime}}$, determined for each windtunnel profile, are summarized in Table III. For practical applications, relations between the length scales $d_{s}$ and $z_{s}$, and morphometric parameters would be very useful. As discussed in Section 2.1, Jackson (1981) interpreted the shear-stress displacement height $d_{s}$ as the average elevation of the surface, which can be estimated by $d_{s}=\bar{h}=\left(A_{p} / A_{T}\right) \bar{H}$. This expression can be reformulated to

$$
d_{s} / \bar{H}=A_{p} / A_{T}=\lambda_{p},
$$

i.e., a linear correlation between the ratio $d_{s} / \bar{H}$ and the plan areal fraction $\lambda_{p}$ is anticipated. In Figure 11a the $d_{s} / \bar{H}$ values for the RdS sampling locations are plotted against $\lambda_{p}$ together with results for the full-scale and wind-tunnel datasets presented in Figure 10 (symbols accordingly). The experimental data agree fairly well with Equation (11). It may be argued that the $d_{s} / \bar{H}$ ratio starts to increase only if $\lambda_{p}$ exceeds the threshold value, which characterizes the transition to skimmingflow regimes. However, the presently available database is not sufficient to prove this concept. 
(a)

(b)
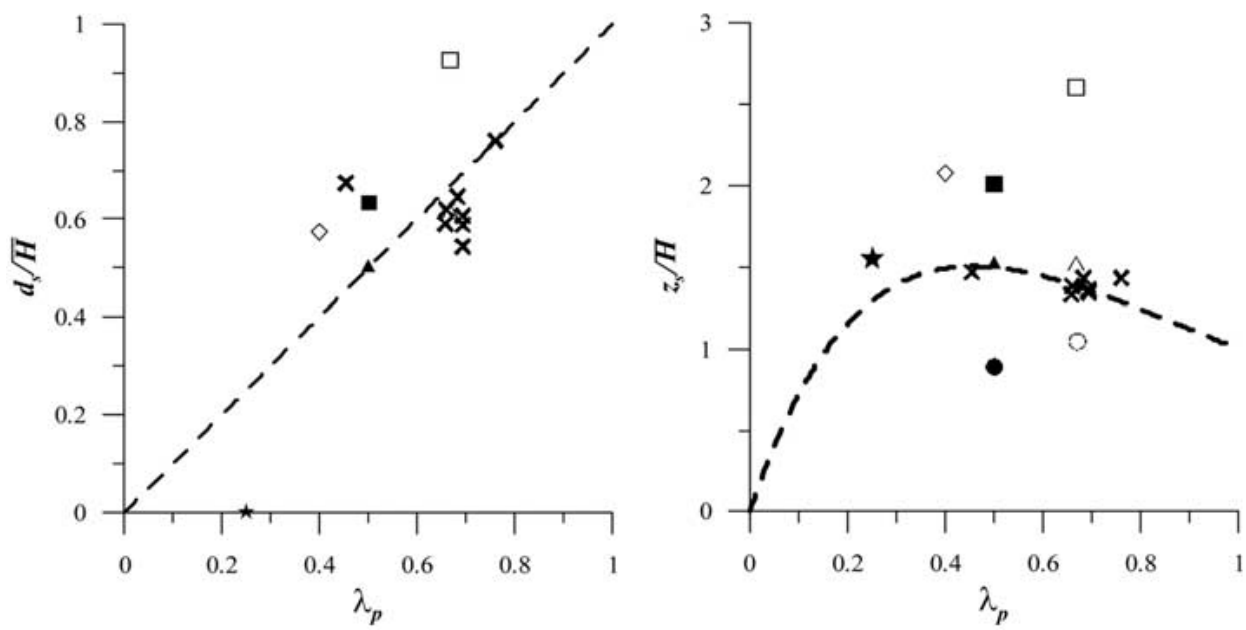

Figure 11. Dependence of (a) shear-stress displacement height, $d_{s}$, and (b) shear-stress peak level, $z_{s}$, on plan areal fraction $\lambda_{p}$. Data from the RdS wind-tunnel study (crosses) and field and wind-tunnel studies published in the literature (symbols according to Figure 10). The dashed lines correspond to Equations (11) and (12), respectively.

Figure $11 \mathrm{~b}$ shows that the relationship between $z_{s} / \bar{H}$ ratios and $\lambda_{p}$ is much less consistent. The $z_{s} / \bar{H}$ results for the RdS study are approximately constant. Meanwhile, a relatively large scatter exists in the data from the field and wind-tunnel studies published in the literature. Although the limited amount of data restricts a more detailed analysis, quasi-physical arguments in analogy to Grimmond and Oke (1999) are used to describe principal characteristics of the relation between $z_{s} / \bar{H}$ and $\lambda_{p}$. If the packing density starts to increase from $\lambda_{p}=0$, where $z_{s} / \bar{H}=0$, the shear-stress peak level will increase and soon be above the average roof level, i.e. $z_{s} / \bar{H}>1$. However, above some threshold value of $\lambda_{p}$ the ratio $z_{s} / \bar{H}$ will start to decrease and if $\lambda_{p}$ approaches unity, so will the ratio $z_{s} / \bar{H}$. In such an extreme case the building tops can be considered as an elevated, smooth surface at the level $\bar{H}$. A general expression for such a relationship can be formulated as $z_{s} / \bar{H}=\left(\lambda_{p}\right)^{c_{1}} \exp \left\{-c_{2}\left(\lambda_{p}-1\right)\right\}$. The dashed, grey line plotted in Figure 11b corresponds to

$$
z_{s} / \bar{H}=\lambda_{p} \exp \left\{-2.2\left(\lambda_{p}-1\right)\right\},
$$

which was determined by fitting the RdS data assuming $c_{1}=1$. Although, Equation (12) will be employed in the further analysis, it is not intended as a general parameterization for $z_{s} / \bar{H}$. In order to establish such a parameterization, more datasets are necessary, and the scatter in Figure $11 \mathrm{~b}$ indicates that additional parameters should be taken into account. We expect that the variability of the building height, $\sigma_{H}$, plays a role and the maximum $z_{s} / \bar{H}$ value may also be influenced by 
the extent of the upstream urban fetch. Figure $11 \mathrm{~b}$ gives some indication that the larger $z_{s} / \bar{H}$ values correspond to field or laboratory situations with a larger urban fetch, but more datasets are needed to verify these results.

In the following discussion $d_{s}$ and $z_{s}$ values, determined by Equations (11) and (12), will be referred as to $d_{s m}$ and $z_{s m}$, where the index $m$ indicates the morphometric estimation approach. Meanwhile, the values directly determined by the shear-stress approximation for each measured profile (see values in Table III) will be referred to as $d_{s}$ and $z_{s}$.

\section{Scaling of Mean Wind Profile}

As a next step, the relation between the scaling parameters derived from the shear-stress parameterizations and parameters of the mean wind profile are studied. For this analysis the relation between the displacement height, $d_{0}$, of the mean wind profile and the length scales $z_{s}$ and $d_{s}$ must be clarified first. This relation is obtained by applying the integral condition (Equation (3)) proposed by Jackson (1981) together with the shear-stress parameterization (Equation 10) presented in the previous section. The mathematical form chosen for the shearstress approximation allows solution of the integral in Equation (3), which then yields

$$
\widehat{d}_{0 i}=\left(d_{0 i}-d_{s}\right)=(2.25-0.25 \exp \{2\}) \hat{z}_{s} \approx 0.4\left(z_{s}-d_{s}\right)
$$

or

$$
d_{0 i} \approx 0.4 z_{s}+0.6 d_{s}
$$

The index $i$ is introduced to denote the estimation of $d_{0}$ by Equation (3). The results for $d_{0 i}$ are given in Table III, and the location of the $d_{0 i}$ levels compared to the $z_{s}$ and $d_{s}$ levels is illustrated in Figure 9 for four profiles.

Using the $d_{0 i}$ values as input parameters in log-law fits of the measured mean wind profiles (see description of the fitting procedure in Section 4), a new set of parameters for the roughness length $z_{0}$ and friction velocity $u_{*}$ can be determined. The values for $z_{0}$ estimated in this way may, on average, be described by the relation:

$$
z_{0 i} \approx 0.12\left(z_{s}-d_{0 i}\right)=0.072\left(z_{s}-d_{s}\right),
$$

i.e., the roughness length, $z_{0}$, can be expressed as a fraction of the distance between the level of the shear-stress peak and the shear-stress displacement height: $z_{0}=$ $f_{0}^{*}\left(z_{s}-d_{s}\right)$. This result illustrates that only the zone of depth $z_{s}-d_{s}$ acts as the roughness layer while the flow is skimming over the lower part of the canopy $(z<$ $d_{s}$ ). The factor $f_{0}^{*} \approx 0.07$ is fairly similar to the rule-of-thumb value $f_{0}=0.1$, 
discussed in Grimmond and Oke (1999) for a simple height-dependent roughnesslength formula $z_{0}=f_{0} \bar{H}$.

Employing the parameterised values $d_{s m}$ and $z_{s m}$ (Equations (11) and (12)) in Equations (13) and (14) morphometric formulae can be derived for $d_{0}$ and $z_{0}$ :

$$
\begin{aligned}
& d_{0 n} / \bar{H} \approx 0.4 \lambda_{p} \exp \left\{-2.2\left(\lambda_{p}-1\right)\right\}+0.6 \lambda_{p}, \\
& z_{0 n} / \bar{H} \approx 0.072 \lambda_{p}\left[\exp \left\{-2.2\left(\lambda_{p}-1\right)\right\}-1\right],
\end{aligned}
$$

which are shown as grey lines in Figure 6. The shapes of the curves are generally reasonable and the magnitudes of the predicted values are within the range of experimental uncertainty. The predicted $d_{0 n} / \bar{H}$ values are at the upper bound and the $z_{0 n} / \bar{H}$ at the lower bound of the range identified as 'physically plausible' by Grimmond and Oke (1999). However, note that Equations (15) and (16) rely on empirical results from the RdS datasets (numerical parameters from the curve fitting) and should, therefore, not be considered as new, universal relationships that can be applied without further considerations to any other urban site.

With $d_{0}$ and $z_{0}$ estimated by $d_{0 n}$ and $z_{0 n}$, the shear stress velocities in Equation (1) can be determined based on the velocity measurement, $u_{\mathrm{ref}}$, in a reference height, $z_{\text {ref }}$, according to:

$$
u_{* r}=\frac{k u_{\mathrm{ref}}}{\ln \left(\frac{z_{\mathrm{ref}}-d_{0 n}}{z_{0 n}}\right)} .
$$

A reference height $z_{\text {ref }}=200 \mathrm{~mm}$ is chosen, but the resulting $u_{* r}$ values are not very sensitive to the particular choice of $z_{\text {ref }}$ (not shown). For $\left(z-d_{0}\right) / z_{0} \geq 10$, which roughly corresponds to $z / d_{0} \geq 1.6$, the wind profiles normalized by the shear stress velocity $u_{* r}$ and the length scales $d_{0 n}$ and $z_{0 n}$ agree well with the log law (Figure 12).

A comparison of $u_{* r}$ values (included in Table III) with the velocity scale, $u_{s}$, shows that, on average, $u_{* r}$ is about $20 \%$ higher than $u_{s}$ (see also Figure 8). Possible reasons for these differences are given below. For example, the turbulent flux component $\overline{v^{\prime} w^{\prime}}$ could not be measured and was therefore ignored in the shearstress analysis. However, in order to explain the $20 \%$ difference, the turbulent flux $\overline{v^{\prime} w^{\prime}}$ must be as high as $\overline{u^{\prime} w^{\prime}}$, which does not seem to be realistic. Furthermore, the attenuation of extreme shear-stress peak values by the parameterization introduced in Section 5 could be too strong. However, the comparison of the different velocity scales in Figure 8 shows that, for the majority of profiles, $u_{* r}$ is even larger than the velocity scales, $u_{f m}$, that are based on the measured shear-stress peak values.

Another way to interpret the differences between $u_{* r}$ and $u_{s}$ is to conclude that the value of the von Kármán constant is about $20 \%$ smaller (i.e., $\kappa \approx 0.32$ ) than the assumed standard value $\kappa=0.4$. This conclusion complies with findings of Oncley et al. (1996) and recent results of Andreas et al. (2002). In both studies, it 


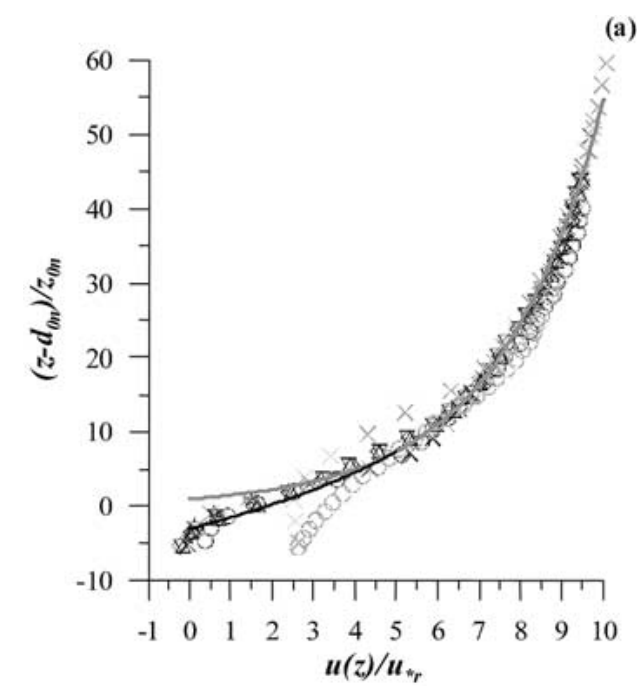

(a)

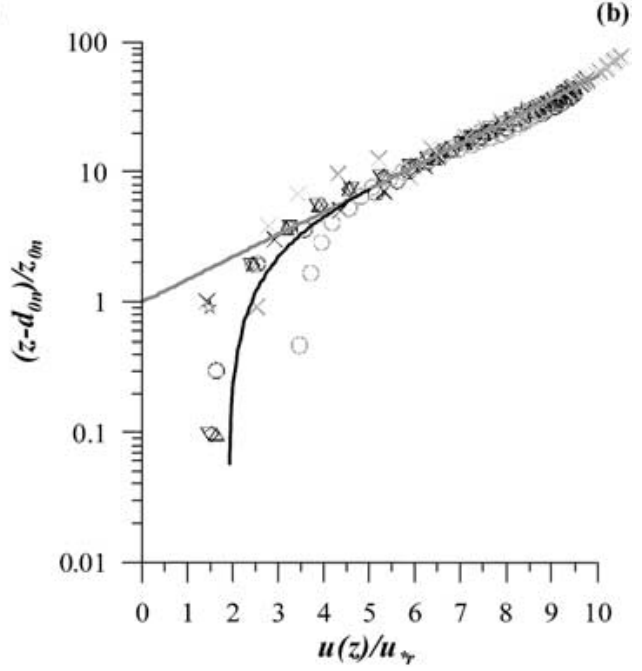

(b)

Figure 12. Comparison of the measured mean wind profiles with the log-law profile (Equation (1), grey line) and a profile for the RSL that is based on a local-scaling approach (Equation (24), black line). The scaling parameters displacement height, $d_{0 n}$, (Equation (15)) and roughness length, $z_{0 n}$, (Equation (16)) are calculated based on the approximated length scales $z_{s m}$ and $d_{s m}$. The shear-stress velocities, $u_{* r}$, (Equation (17)) are based on a velocity value, $u_{\mathrm{ref}}$, measured in the reference height, $z_{\text {ref }}=200 \mathrm{~mm}$. Plot (b) shows the same as plot (a) but on a semi-log scale.

was found that the von Kármán constant appears not to be constant but a function of the roughness Reynolds number, $\operatorname{Re}_{*}=\left(u_{*} z_{0}\right) / v$. Oncley et al. (1996) note that $\kappa$ decreases from the value 0.4 for smooth surfaces to the value $\approx 0.34$ for rough surfaces. Andreas et al. (2002) observed an even stronger reduction and presented a best fit to their data given by

$$
\kappa=-0.0455 \ln \left(\operatorname{Re}_{*}\right)+0.514
$$

Based on the average $u_{*}$ and $z_{0}$ values (see Table III) the roughness Reynolds number $\mathrm{Re}_{*}$ for the investigated urban area can be estimated to be approximately 270 , for which Equation $(18)$ results in $\kappa \approx 0.26$. These estimates show that $\kappa \approx 0.32$ falls within the range of values that can be expected for rough urban areas. However, for the further analysis and discussion we decided to adhere to the assumption of a constant $\kappa$ with a value of 0.4 , since the dependence of $\kappa$ on $\mathrm{Re}_{*}$ needs further verification. As an example, the problem of the definition of $u_{*}$ over very rough surfaces, where a constant flux layer is often not established, has only been marginally addressed in previous studies. The wind-profile analysis undertaken by Andreas et al. (2002) provides only the slope parameter $u_{*} / \kappa$, and the definition of $u_{*}$ is, therefore, of crucial importance for the determination of the von Kármán constant. Furthermore, the concept of a non-constant $\kappa$ cannot easily be applied in practice as long as a sound functional dependence on $\mathrm{Re}_{*}$ does 
not exist. The variation of the estimated values discussed earlier in this paragraph shows that this is presently not the case.

MacDonald et al. (2002) also found poor agreement between $u_{*}$ values determined by fitting the log law to mean wind profiles and turbulent shear stresses or surface shear stress measured by a drag plate. They discuss that, in most laboratory studies, the fetch is too short to reproduce a boundary layer that is in equilibrium with the underlying urban surface. They argue that for such conditions the non-dimensional shear,

$$
S=\frac{\kappa\left(z-d_{0}\right)}{u_{*}} \frac{\partial u}{\partial z},
$$

is significantly larger than one and that the slope parameter of the mean wind profiles becomes, therefore, $S u_{*} / \kappa$ (instead of $u_{*} / \kappa$ ), which would explain why $u_{*}$ estimates based on log-profile fits typically exceed shear-stress based $u_{*}$ estimates. However, they assume in their analysis that $\kappa$ is constant and equals 0.4. Following the discussion in the previous paragraph the deviation of $S$ from unity could also be explained by $\kappa<0.4$.

The previous analysis and discussion has shown that the measured mean urban wind profiles can be fairly well approximated by the log law above the level $z=$ $1.6 d_{0}$. However, a parameterization of the mean wind profile inside the RSL is still lacking. Following the concept of Rotach (2001), it could be argued that a local, height-dependent scaling velocity defined according to

$$
u_{s l}=\sqrt{-\overline{u^{\prime} w^{\prime}}(z)}
$$

applies inside the RSL. If it is further assumed that

$$
\frac{\mathrm{d} u}{\mathrm{~d} z}=\frac{u_{s l}}{\alpha\left(z-d_{s}\right)}
$$

is valid inside the RSL, a velocity profile below the level $z=z_{s}$ can be formulated applying the proposed shear-stress parameterization (Equation (10)),

$$
u_{\mathrm{RSL}}(z)=\frac{u_{s l}}{\alpha} \exp \left\{1-\left(\frac{z-d_{s}}{z_{s}-d_{s}}\right)\right\}+C .
$$

Expressions for the integration constant $C$ and proportionality factor $\alpha$ can be found by matching the RSL profile (Equation (21)) and log-law profile (Equation (1)) at the level $z=z_{s}$. As first matching condition, the velocity values must be equal at this level, i.e.:

$$
-\frac{u_{s l}}{\alpha}+C=\frac{u_{*}}{\kappa} \ln \left(\frac{z_{s}-d_{0}}{z_{0}}\right) .
$$


As a second condition, the equality of the velocity gradients has been postulated:

$$
\frac{u_{s l}}{\alpha\left(z_{s}-d_{s}\right)}=\frac{u_{*}}{\kappa\left(z_{s}-d_{0}\right)} .
$$

Using these two relationships, together with Equations (13) and (14), the RSL profile can be expressed as

$$
u_{\mathrm{RSL}}(z)=\frac{u_{*}}{0.6 \kappa}\left[1-0.6 \ln (0.12)-\exp \left\{0.6-0.072\left(\frac{z-d_{0}}{z_{0}}\right)\right\}\right] .
$$

This velocity profile agrees fairly well with the measured data (Figure 12). Inside the RSL, the local scaling approach (Equation (24)) is clearly an improvement compared to the log law. However, the limitations of gradient-diffusion models discussed by MacDonald (2000) apply also to the local scaling approach. Street-canyon type (or skimming-flow) wind profiles, which are characterized by approximately constant and typically negative velocity values inside the canopy, cannot be predicted.

\section{Conclusions}

The mean wind profiles above the urban structure can be well approximated by the log law, despite a typically rather shallow ISL. Inside the RSL a local scaling approach results in good agreement between measured and calculated mean wind profiles. A proposed scaling and parameterization for the shear-stress profiles describes wind-tunnel and full-scale data well.

For future applications, it seems important to incorporate a shear-stress displacement height, $d_{s}$, into urban boundary-layer concepts. Furthermore, the height, $z_{s}$, where the shear-stress peak is observed has been proved to be an important length scale in the UBL. The length scales of the mean ISL wind profile, $z_{0}$ and $d_{0}$, can both be described by a linear correlation with $z_{s}-d_{s}$, which indicates that $z_{s}-d_{s}$ corresponds to the layer where the flow feels the disturbances induced by the roughness elements. Future research is necessary in order to verify the proportionality constants. However, the analysed correlations between the length scales $z_{s}, d_{s}$ and morphometric parameters are promising, and the correlation between $d_{s}$ and surface morphometry appears to be straightforward. The relation $d_{s} / \bar{H}=\lambda_{p}$ that follows from the arguments presented by Jackson (1981) describes the experimental results fairly well. A parameterization for $z_{s} / \bar{H}$, based on quasi-physical reasoning, works well for the RdS data, but requires further verification. Realistic values could be predicted for the wind-profile parameters $z_{0}$ and $d_{0}$ based on the morphometric parameterizations for $z_{s}$ and $d_{s}$.

Overall, it can be concluded that the length scales of the proposed shear-stress parameterization are well described by the proposed morphometric methods and 
can be successfully used to estimate the wind-profile parameters $d_{0}$ and $z_{0}$. For the friction velocity, $u_{*}$, the best estimation method appears then to be Equation (17), if one wants to describe the mean ISL wind profile with the log law (Equation (1)). This method requires morphometric $d_{0}$ and $z_{0}$ estimates and wind velocity measurements at a reference height. All tested methods to derive $u_{*}$ from the measured or parameterized shear-stress profiles have been less accurate. Measured shear-stress peak values are strongly affected by local flow disturbances and result, therefore, in unrealistic variations of $u_{*}$. Such fluctuations can be successfully avoided if peak values of the parameterized shear-stress profiles are considered, but the absolute values of these velocity scales are too low. The latter discrepancies might be related to the fact that the flow is not in equilibrium with the underlying surface. The $u_{*}$ estimates based on log-profile fits should then be interpreted as the scaling velocity for the mean wind profile rather than an estimate of a true friction velocity. Another reason for the discrepancies may relate to a deviation of the von Kármán constant from $\kappa=0.4$ over rough urban surfaces, but the present state of knowledge does not permit reliable estimates of the variations of $\kappa$. Consequently, the assumption of $\kappa=0.4$, and the combination of morphometric estimation methods for $d_{0}$ and $z_{0}$ with Equation (17), appears to be the most reliable and straightforward procedure to determine the mean wind-profile parameters.

\section{Acknowledgements}

The work was supported by the European commission and the Swiss Ministry of Education and Science (grant 97.0136) within the TMR-project TRAPOS. We are also grateful to the co-operation with the URBCAP Nantes-99 campaign. Special thanks go to Harald Deutsch, Armin Reinsch, Alexis Madrange and Evgeni Fedorovich, who were responsible for planning and conducting the wind-tunnel experiments at the Institute of Hydromechanics at the University of Karlsruhe, Germany, and to Bob Conzemius for assisting in preparation of the manuscript.

\section{References}

Andreas, E. L., Claffey K. J., Fairall, C. W., Guest, P. S., Jordan, R. E., and Persson, P. O. G.: 2002, 'Evidence from the Atmospheric Surface Layer that the von Kármán Constant Isn't', in Proceedings of the 15th Symposium on Boundary Layers and Turbulence, Wageningen, The Netherlands, 15-19 July 2002.

Brown, M. J., Lawson, R. E., Descroix, D. S., and Lee, R. L.: 2000, 'Mean Flow and Turbulence Measurements around a 2-D Array of Buildings in a Wind Tunnel', in 11th Conf. on Appl. of Air Poll. Met., Long Beach, CA, U.S.A., January 2000.

Cheng, H. and Castro, I. P.: 2002, 'Near Wall Flow over Urban-Like Roughness', Boundary-Layer Meteorol. 104, 229-259.

Christen, A., Vogt, R., Rotach, M. W., and Parlow, E.: 2002, 'First Results from BUBBLE I: Profiles of Fluxes in the Urban Roughness Sublayer', in Proceedings of the 4th Symposium on the Urban Environment, Norfolk, VA, U.S.A., 20-24 May 2002. 
Elliott, W. P.: 1958, 'The Growth of the Atmospheric Internal Boundary Layer', Trans. Amer. Geophys. Union 39, 1048-1054.

Feigenwinter, C., Vogt, R., and Parlow, E.: 1999, 'Vertical Structure of Selected Turbulence Characteristics above an Urban Canopy', Theor. Appl. Climatol. 62, 51-63.

Garratt, J. R.: 1990, 'The Internal Boundary Layer - A Review', Boundary-Layer Meteorol. 50, $171-203$.

Grimmond, C. S. B. and Oke, T. R.: 1999, 'Aerodynamic Properties of Urban Areas Derived from Analysis of Surface Form', J. Appl. Meteorol. 38, 1262-1292.

Hussain, M. and Lee, B. E., 1980: 'A Wind Tunnel Study of the Mean Pressure Forces Acting on Large Groups of Low Rise Buildings', J. Wind Eng. Ind. Aerodyn. 6, 207-225.

Jackson, P. S.: 1981, 'On the Displacement Height in the Logarithmic Velocity Profile', J. Fluid Mech. 111, 15-25.

Kaimal, J. C. and Finnigan, J. J.: 1994, Atmospheric Boundary Layer Flows. Their Structure and Measurement, Oxford University Press, U.K., 289 pp.

Kastner-Klein, P.: 1999, Experimentelle Untersuchung der strömungsmechanischen Transportvorgänge in Straßenschluchten, Dissertation, Universität Karlsruhe, Germany.

Kastner-Klein, P., Fedorovich, E., and Rotach, M. W.: 2001, 'A Wind Tunnel Study of Organised and Turbulent Air Motions in Urban Street Canyons', J. Wind Eng. Ind. Aerodyn 89, 849-861.

Louka, P.: 1999, Measurements of Airflow in an Urban Environment, Ph.D. Thesis, University of Reading, U.K.

Louka, P., Belcher, S. E., and Harrsion R.G., 2000: 'Coupling between Air Flow in Streets and the Well-Developed Boundary Layer Aloft', Atmos. Environ. 34, 2613-2621.

MacDonald, R.W.: 2000, 'Modelling the Mean Velocity Profile in the Urban Canopy Layer', Boundary-Layer Meteorol. 97, 25-45.

MacDonald, R. W., Carter Schofield, S., and Slawson, P. R.: 2002, 'Turbulence Statistics in the Developing Urban Boundary-Layer', in Proceedings of the 4th Symposium on the Urban Environment, Norfolk, VA, U.S.A., 20-24 May 2002.

MacDonald, R. W., Griffiths, R. S., and Hall D. J.: 1998, 'An Improved Method for the Estimation of Surface Roughness of Obstacle Arrays', Atmos. Environ. 32, 1857-1894.

Martilli, A., Clappier, A., and Rotach, M.W.: 2002, 'An Urban Surface Exchange Parameterisation for Mesoscale Models', Boundary-Layer Meteorol. 104, 261-304.

Oikawa, S. and Meng, Y: 1995, 'Turbulence Characteristics and Organized Motion in a Suburban Roughness Sublayer', Boundary-Layer Meteorol. 74, 289-312.

Oncley, S. P., Friehe, C. A., Larue, J. C., Businger, J. A., Itsweire, E. C., and Chang, S. S.: 1996, 'Surface-Layer Fluxes, Profiles and Turbulence Measurements over Uniform Terrain Under Near-Neutral Conditions', J. Atmos. Sci. 53, 1029-1044.

Panofsky, H. A. and Dutton, J. A.: 1984, Atmospheric Turbulence: Models and Methods for Engineering Applications, Wiley-Interscience, New York, 397 pp.

Pendergrass, W. and Arya, S. P. S.: 1984, 'Dispersion in Neutral Boundary Layer over a Step Change in Surface Roughness - I. Mean Flow and Turbulence Structure', Atmos. Environ. 18, 1267-1279.

Plate, E. J.: 1995, 'Urban Climates and Urban Climate Modelling: An Introduction', in J. E. Cermak et al. (eds.), Wind Climate in Cities, Kluwer Academic Publishers, Boston/Dordrecht, pp. 23-29.

Rafailidis, S.: 1997, 'Influence of Building Areal Density and Roof Shape on the Wind Characteristics above a Town', Boundary-Layer Meteorol. 85, 255-271.

Raupach, M. R.: 1980, 'A Wind-Tunnel Study of Turbulent Flow Close to Regularly Arrayed Roughness elements', Boundary-Layer Meteorol. 18, 373-397.

Raupach, M. R., Antonia R. A., and Rajagopalan S.: 1991, 'Rough-Wall Turbulent Boundary Layers', Appl. Mech. Rev. 44, 1-25.

Rotach, M. W.: 1993a, 'Turbulence Close to a Rough Urban Surface, Part I: Reynolds Stress', Boundary-Layer Meteorol. 65, 1-28. 
Rotach, M. W.: 1993b, 'Turbulence Close to a Rough Urban Surface, Part II: Variances and Gradients', Boundary-Layer Meteorol. 66, 75-92.

Rotach, M. W.: 1995, 'Profiles of Turbulence Statistics in and above an Urban Street Canyon', Atmos. Environ. 29, 1473-1486.

Rotach, M. W.: 1999, 'On the Influence of the Urban Roughness Sublayer on Turbulence and Dispersion', Atmos. Environ. 33, 4001-4008.

Rotach, M. W.: 2001, 'Simulation of Urban-Scale Dispersion Using a Lagrangian Stochastic Dispersion Model', Boundary-Layer Meteorol. 99, 379-410.

Roth M., 2000: 'Review of Atmospheric Turbulence over Cities', Quart. J. Roy. Meteorol. Soc. 126, 941-990.

Theurer, W.: 1999, 'Typical Building Arrangements for Urban Air Pollution Modelling', Atmos. Environ. 33, 4057-4066.

Vachon, G., Louka, P., Rosant, J-M., Mestayer, P., and Sini, J-F.: 2001, 'Measurements of TrafficInduced Turbulence within a Street Canyon during the Nantes '99 Experiment', J. Water Air Soil Poll.: Focus 2(5-6), 127-140.

Vachon, G., Rosant, J-M., Mestayer, P., and Sini, J-F.: 1999, 'Measurements of Dynamic and Thermal Field in a Street Canyon, URBCAP Nantes 99', in Proceedings of the 6th International Conference on Harmonisation within Atmospheric Dispersion Modelling, October 11-14, Rouen, France. 